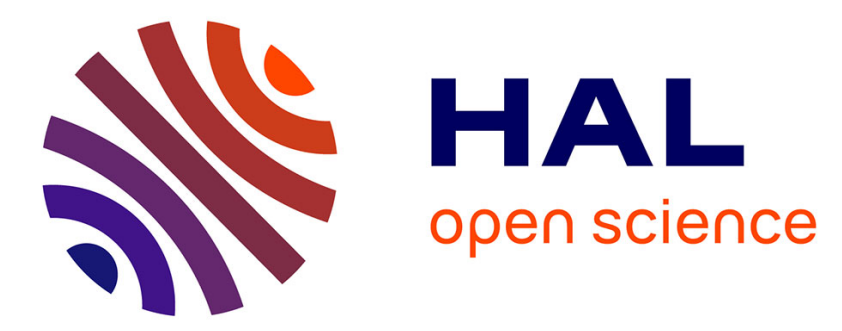

\title{
Multivariate Statistical Analysis for Exploring Road Crash Related Factors in the Franche-Comté region of France
}

Cécile Spychala, Joël Armand, Clément Dombry, Camelia Goga

\section{To cite this version:}

Cécile Spychala, Joël Armand, Clément Dombry, Camelia Goga. Multivariate Statistical Analysis for Exploring Road Crash Related Factors in the Franche-Comté region of France. 2021. hal-02735348v2

\author{
HAL Id: hal-02735348 \\ https://hal.science/hal-02735348v2
}

Preprint submitted on 27 Apr 2021

HAL is a multi-disciplinary open access archive for the deposit and dissemination of scientific research documents, whether they are published or not. The documents may come from teaching and research institutions in France or abroad, or from public or private research centers.
L'archive ouverte pluridisciplinaire HAL, est destinée au dépôt et à la diffusion de documents scientifiques de niveau recherche, publiés ou non, émanant des établissements d'enseignement et de recherche français ou étrangers, des laboratoires publics ou privés. 


\title{
Multivariate Statistical Analysis for Exploring Road Crash Related Factors in the Franche-Comté region of France
}

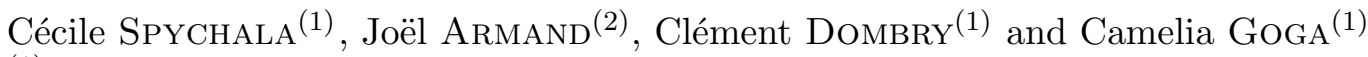 \\ (1) LMB, Université de Bourgogne Franche-Comté, Besançon, FRANCE \\ (2) Gendarmerie Nationale de Besançon, Besançon, FRANCE \\ cecile.spychala@univ-fcomte.fr, joel.armand@gendarmerie.interieur.gouv.fr \\ clement.dombry@univ-fcomte.fr, camelia.goga@univ-fcomte.fr
}

\section{ARTICLE HISTORY}

Compiled April 27, 2021

\begin{abstract}
Understanding and modelling road crash data is crucial in fulfilling safety goals by helping national authorities to take necessary measures to reduce crash frequency and severity. This work aims at giving a multivariate statistical analysis of road crash data from the French region of Franche-Comté with special attention to road crash gravity. The first step for this multivariate analysis was to perform Multiple Correspondence Analysis in order to assess associations between the road crash injury and several important accident-related factors and circumstances. Log-linear models are used next in order to detect associations between road crash severity and related factors such as alcohol/drug consumption or spatial crash locations. The effects of each factors have been also evaluated on the road crash gravity by using ordinal logistic regression. Data used in this study are extracted from BAAC files, the French census of road crashes.
\end{abstract}

Key words: geometric data analysis, multiple correspondence analysis, ordinal logistic regression, log-linear model, road crash severity.

\section{Introduction}

Over the last decade, the number of road crashes has continuously been decreasing in France. Indeed, 61224 accidents have been recorded in 2017 instead of 58352 in 2018, a decrease of 4,7\% (ONISR, 2019). However, road accidents still happen and important efforts and means are developed to prevent them. Among these, modern statistical methods are efficient prevention tools used to describe and model accident data. This paper is concerned about road accidents that occurred in the Franche-Comté region of France (see FIG. 1). This region from the east of France is split up into four departments called Doubs, Jura, Haute-Saône and Teritoire de Belfort. Regarding to the mortality rate from 2017 to 2018, this rate has globally decreased for this region. However, the situation is quite different within each department. Indeed, the death rate has increased by 3\% from 2017 in the Doubs department while it has decreased in the Haute-Saône, Jura and Territoire de Belfort departments by 45\%, 65\% and 
respectively by 50\% (ONISR, 2019). Understanding and modelling accident data is crucial in fulfilling safety goals by helping national authorities to undertake necessary measures to reduce crash frequency and severity.

This paper focuses on accidents in Franche-Comté involving casualties. An accident refers to a road crash with casualty needing hospital care and can involve several cars and several people. One of the main goals of the National Gendarmerie of Besançon (Doubs, France) is to reduce the number of accidents in Franche-Comté. More precisely, the National Gendarmerie of Besançon plans to be able in the near future to anticipate road crashes by using time and spatial modelling of accident data. This study aims at giving a multivariate statistical analysis of the road crashes in Franche-Comté. A first multivariate descriptive study of French accident data was conducted by Bièvre (2017) in an unpublished technical report. We intend in this work to give a deeper analysis of Franche-Comté accident data.

The main goal of this research work is to explain the variable giving the severity or the gravity of the accidents by using several covariates such as spatial location, time period, weather conditions, road type, alcohol/drug consumption... Our multivariate statistical analysis starts with a Multiple Correspondence Analysis (MCA). The MCA as suggested by Benzécri (Benzécri, 1973, 1982) is the generalization of the Correspondence Analysis (CA) for analyzing jointly more than two categorical variables. This method is widely used in categorical data analyses because it allows detecting similarities between individuals and assessing associations between categories. Geometric representations of data clouds in smaller dimension spaces allow identifying clusters of similar individuals and of associated categories or variables. Many applications of MCA and related methods in various fields such as social, demographic, economic are given in Greenacre and Blasius (2006). The goal here is to determine the accident factors mostly related to road crash severity. In the literature concerning the accident analysis and prevention, several studies used MCA in various contexts but different from our framework. For example, Das and Sun (2015) used eight years of pedestrian crash data and MCA to identify key associations between risk factors and Das and Sun (2016) used MCA to identify crash-prone factors producing fatal run-off-road crashes; Das et al. (2018) investigated the wrong way driving crash patterns by using MCA while Fort et al. (2019) tried to explain working conditions and risk exposure of employees whose occupations require driving on public roads.

The MCA analysis conducted on the Franche-Comté accident data set allows us to identify several variables associated with the road crash severity. A more in-depth analysis of these variables is next considered by log-linear modelling (Agresti, 2013). The log-linear model belongs to the class of generalized linear model (McCullagh and Nelder, 1989). In the case of categorical data, the cell counts of the contingency table are modeled by a Poisson distribution and a log link function is used for the mean. More precisely, the log-linear model specifies how the expected counts depend on the levels of the categorical variables and it allows to quantify the associations and interactions between those variables. Unlike MCA, log-linear models allow getting insight into complex dependence patterns such as conditional or marginal dependence which may exist between several categorical variables. In our framework, we will use log-linear models in order to detect conditional or marginal associations between road crash severity and other variables such as alcohol/drug consumption and spatial location. In a similar way, Abdel-Aty et al. (1998) used log-linear models to explain associations between the driver age and several important factors and circumstances related to the accident. Also, Yannis et al. (2005) performed a log-linear analysis in order to test the significance of first- and second-order effects among various combinations of driver age 
and engine size categories in relation to two-wheeler accident severity and at-fault risk rates. Then, Abdel-Aty and Abdelwahab (2000) used log-linear models to investigate whether there are associations between the different driver characteristics and alcohol involvement and also in order to identify the high-risk group within each driver factor.

Finally, we propose ordinal logistic regression (Agresti, 2013) to model the gravity level probabilities as a function of explicative covariates such as alcohol/drug consumption, time period and spatial locations. This method widely used in accident data analysis is a popular supervised learning method for analyzing dependencies between a binary or multiclass response categorical variable and several explanatory variables. It allows in particular to separate and identify the effects of each explanatory variable on the response variable. Rezapour and Ksaibati (2018) used ordinal logistic regression to investigate the contributory factors that increased the odds of severe single-truck and multiple-vehicle crashes such as characteristics related to driver or vehicle for instance. Then, Mekonnen (2018) has also performed ordinal logistic regression in order to identify the risk factors among driver age, speed record or alcohol consumption for example for severity levels of road traffic accident.

The paper is structured as follows. We first describe in Section 2 our data set as well as the analysis methods: MCA is described briefly in Section 2.2.1, log-linear modelling in Section 2.2.2 and ordinal logistic regression in Section 2.2.3. Section 3 contains the main results of our study and, lastly, Section 4 concludes and proposes several recommendations and perspectives.

\section{Material and methods}

\subsection{Franche-Comté accident data}

Data used in this study concern the Franche-Comté road crashes between 2005 to 2018 which are extracted from the French national analysis bulletin of road traffic injury accidents called BAAC ${ }^{2}$ (Bulletin d'Analyse des Accidents Corporels). The BAAC data are filled in by the security forces present on the accident scene and next, data are treated, analyzed and put online by the national interdepartmental observatory of road safety (Observatoire National Interministériel de la Sécurité Routière).

The BAAC files contained more than 50 variables from which 15 new categorical variables have been created and/or reclassified. The Franche-Comté accident dataset has 11776 casualties registered in 4950 accidents. The study focuses only on the accident itself and not on each casualties. The region Franche-Comte is situated in the east of France and neighboring Switzerland as we can see from Fig. (1). The counties situated on the west of Franche-Comté are mountainous and entirely deserved by national and departmental roads. A daily intensive border activity between Switzerland and France is also present in these counties.

The analysis emphasizes the accident severity, denoted by type_acc, classified into three ordered levels: "slight_safe", "serious" and "fatal". An accident is considered as "slight_safe" (11,47\% of accidents) if all passengers were safe or had minor injuries; the label "serious" was attributed to accidents involving at least one casualty needing hospital care for more than 24 hours $(69,82 \%$ of accidents) and lastly, an accident is considered as "fatal" $(18,71 \%$ of accidents) if at least one casualty involved died.

The alcohol/drug consumption by car drivers is one of the main accident causes and has a great impact on their severity. The categorical variable substance describing the

\footnotetext{
${ }^{2}$ The reader can find the BAAC open data on the government website https://www.data.gouv.fr/fr/
} 


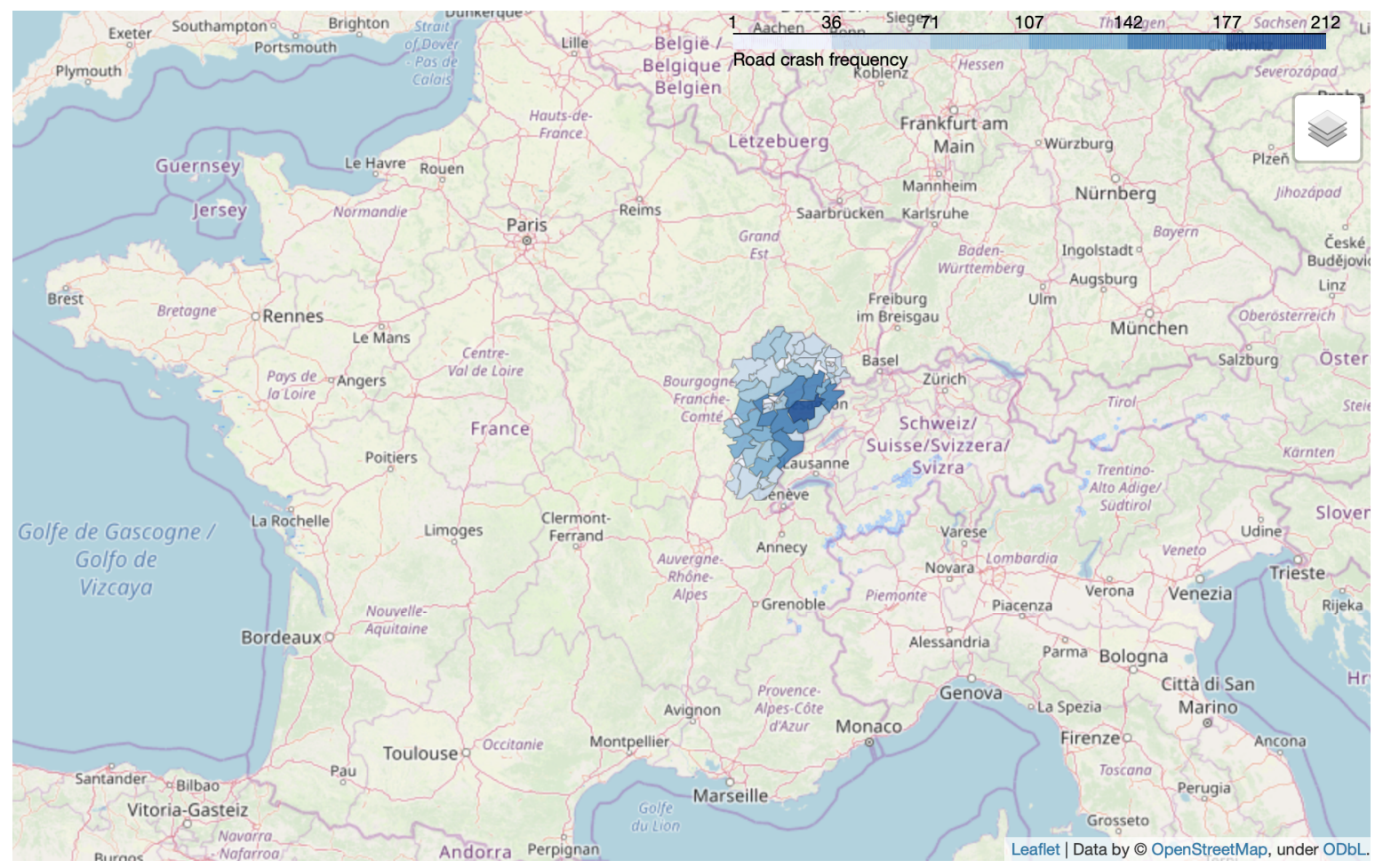

FIG 1.: France map with road crash frequency of Franche-Comté region. Each small division corresponds to a canton.

alcohol/drug consumption by the drivers involved in a road crash has the following levels:

- "alcohol_drug" when at least one of the involved drivers has consumed both alcohol and drugs (2.69\%);

- "drug" when at least one of the involved drivers has consumed drugs $(2.73 \%)$;

- "alcohol" when at least one of the involved drivers has consumed alcohol but not drugs $(16.22 \%)$;

- "none" is associated with accidents involving only sober drivers (78.36\%).

If the accident involves only one driver, the variable substance concerns the unique driver.

As mentioned above, the goal of this study is the statistical analysis of accidents and an accident may involve several drivers and casualties. Variables such as age or sex refer to individuals and are not straightforward to recode for an accident involving several persons. For this reason, age or sex do not appear in our multivariate analysis.

In order to give a more thorough statistical analysis, we considered further 7 categorical variables giving supplementary information about the weather, the type of the road and of the collision:

- weather with two categories: normal and other kind (such as rainy, cloudy or snowy weather);

- area with two categories: unurban and urban;

- intersection with two categories: intersection and out_of_intersection;

- obstacle corresponding to a mobile obstacle with four categories: vehicle, pedestrian, other_kind (such as animals) and none; 
- shape_road with two categories: curve and straight;

- collision with three categories: usual (such as frontal or rear-end collisions), other_kind and none;

- type_road with five categories: communal, departmental, national, highway and other_kind (such as parking).

The above variables will be denoted in the paper as general features.

In order to conduct the temporal analysis of the Franche-Comté road crashes, we used the following categorical variables related to the time period when the accident occurred, denoted in the rest of the paper as temporal features:

- season with four categories: spring, summer, autumn and winter;

- week with two categories: weekday and week_end;

- daytime with two categories: day and night;

- time with five categories: 7am_10am, 11am_3pm, 4pm_7pm, 8pm_11pm and midnight_6am. Note that the category 7am_10am means from 7:00 am to 10:59 am. It is also the case for the other categories of time.

In our accident data, each accident is located by the commune (town or village) and the department (Doubs, Jura, Haute-Saône, Territoire de Belfort) where the accident took place. The variable commune was used to build the variable canton (district) by regrouping the 1176 communes into 50 cantons. In fact, the region of Franche-Comté is splitted up into 62 cantons, however, some cantons have been grouped together as for instance "Belfort-1", "Belfort-2" and "Belfort-3" into "Belfort". This reclassification allows to smooth the variability of cantons categories. Hence, Jura department is divided into 15 cantons, Haute-Saône and Doubs both into 14 cantons and Territoire de Belfort into 7 cantons. The categorical variables department and canton have been used for the spatial analysis, whereas the variable commune was dropped due to too many categories. We give in FIG. (1) the division of Franche-Comté into cantons with their road crash frequencies; Jura department is the department with the highest road crash frequency. These two variables will be denoted in the rest of the paper as spatial features.

TAB 1 gives the cross-tabulation of the accident severity (type_acc) with the different categorical variables. 


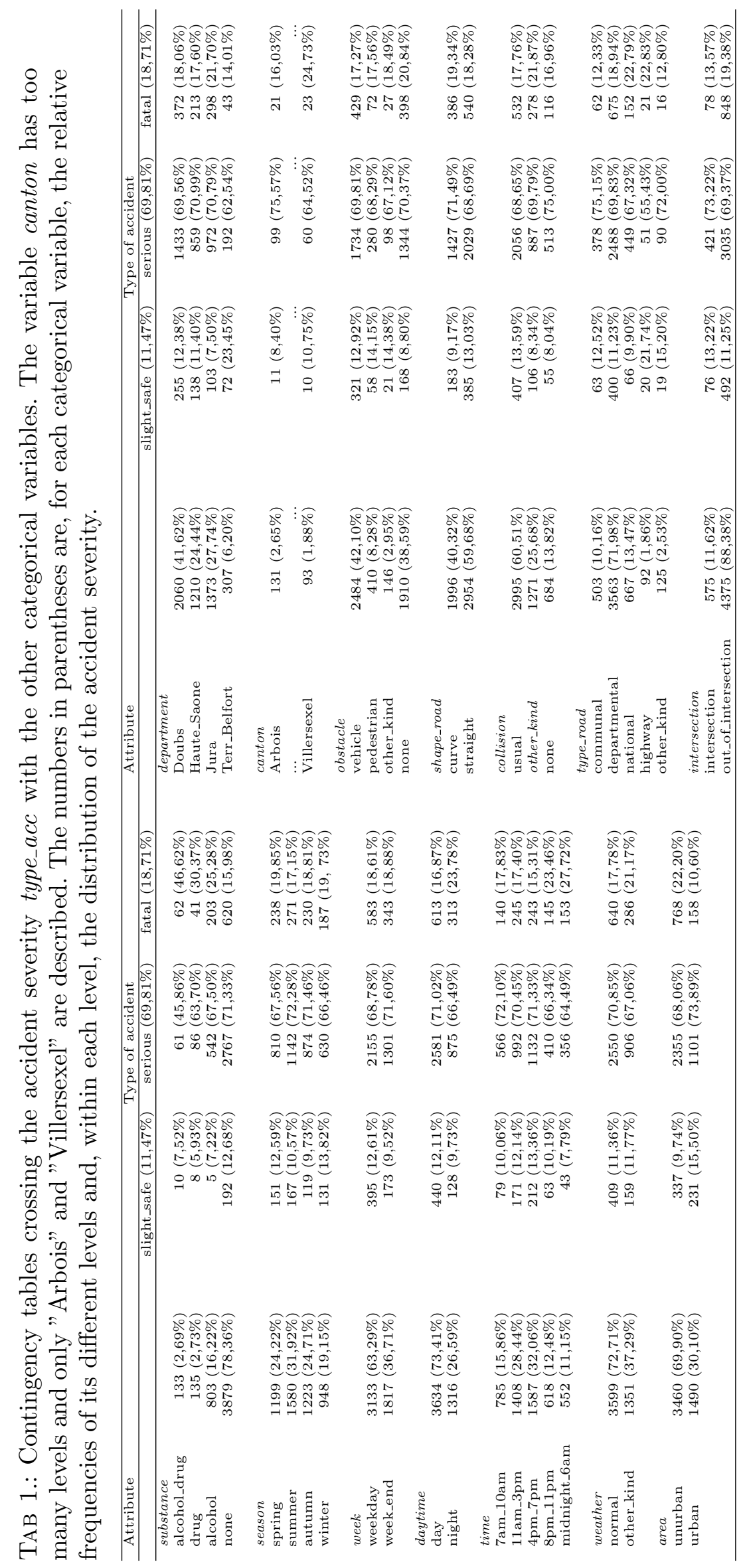




\subsection{Statistical analysis}

\subsubsection{Multiple Correspondence Analysis}

The Multiple Correspondence Analysis (MCA) is an efficient unsupervised method for exploring multivariate categorical data. The aim of MCA is to study the similarities between the individuals, to assess the relationships between the variables and to examine the associations between the categories. For a thorough description of MCA as well as of related methods, the reader is referred to the book of Greenacre and Blasius (2006). This method allows, if appropriate, to corroborate a strong link between categorical variables. In some cases, MCA enables to cluster categories and to reduce the data dimension allowing multivariate data to be analyzed more easily. Indeed, a graphical representation of individuals and variables is built in an orthogonal system similarly as in Correspondence Analysis (CA). This statistical tool is powerful for understanding, visualizing and simplifying the data.

MCA can be derived in several ways. One way is to apply CA on the indicator matrix $\mathbf{X}=\left[\begin{array}{llll}\mathbf{X}_{1} & \mathbf{X}_{2} & \ldots & \mathbf{X}_{p}\end{array}\right]$ derived from the original data Individuals $\times$ Categorical variables of $p$ categorical variables recorded on $n$ individuals. Each indicator matrix $\mathbf{X}_{j}$ is obtained by column concatenation of $K_{j}$ dummy variables where $K_{j}$ is the number of categories of the $j$ th categorial variable, $j=1, \ldots, p$. Hence, $\mathbf{X}$ is a respondentsby-categories matrix having $n$ rows, corresponding to individuals, and $K=\sum_{j=1}^{p} K_{j}$ columns, corresponding to variable categories. An element of this table, denoted by $x_{i k}$, is equal to 1 if the individual $i$ has the category $k$ and 0 otherwise, $i=1, \ldots, n$ and $k=1, \ldots, K$. The indicator matrix $\mathbf{X}$ has row sums equal to the constant $p$ and column sums equal to $n_{k}$, the marginal frequency of the $k$ th category, namely the number of individuals having the category $k$.

This kind of data implies the study of three kinds of objects: the individuals, the variables but also their categories. The scheme of MCA is to compare individuals and evaluate variables characteristics by providing row typologies, column typologies and the relationships between these typologies (Escofier and Pagès, 2008).

From a technical point of view, MCA uses as CA the $\chi^{2}$ distance in order to assess similarity or dissimilarity between different columns or lines contained in $\mathbf{X}$. The indicator matrix $\mathbf{X}$ is transformed in order to obtain row profiles by dividing each element of a row by the row frequency as well as column profiles by dividing each element of a column by its frequency. In the case of MCA, row and column profiles are very simple. The $i$ th row profile is given by $\left(x_{i k} / p\right)_{k=1}^{K}$ : the elements of a row profile have only zero and $1 / p$ values, the non-zero value being recorded if the individual $i$ possesses the category $k$. So, row profiles will be different only for $i$ th and $i^{\prime}$ th individuals having mismatching category levels. The $k$ th column profile is given by $\left(x_{i k} / n_{k}\right)_{i=1}^{n}$ : the elements of the column profile are zero and $1 / n_{k}$ values.

The $\chi^{2}$-distance between two individuals $i$ and $i^{\prime}$ is a weighted sum of squared distances between the $i$ th and $i^{\prime}$ th row profiles with weights given by the inverse of the average row profile given by $\left(n_{k} / n p\right)_{k=1}^{p}$ :

$$
d_{i, i^{\prime}}^{2}=\sum_{k=1}^{K} \frac{n p}{n_{k}}\left(\frac{x_{i k}}{p}-\frac{x_{i^{\prime} k}}{p}\right)^{2}=\frac{n}{p} \sum_{k=1}^{K} \frac{\left(x_{i k}-x_{i^{\prime} k}\right)^{2}}{n_{k}}, \quad 1 \leq i, i^{\prime} \leq n .
$$

Hence, the terms from the above sum will be all zero for coincident zero values and coincident $1 / p$ values meaning that these squared differences will not contribute to the distance measure. Only differences between noncoincident categories will contribute 
to the distance $d_{i, i^{\prime}}^{2}$ and this contribution is proportional to $(1 / p)^{2}$ with weight equal to the inverse of the marginal frequency $n_{k}$. The $\chi^{2}$ distance between row profiles can be interpreted as a weighted mismatching dissimilarity coefficient: small distance $d_{i, i^{\prime}}$ means that individuals $i$ and $i^{\prime}$ have many categories in commun, so they are very similar and on the contrary, large distance $d_{i, i^{\prime}}$ means that $i$ and $i^{\prime}$ have few categories in commun, so they are very different. Moreover, a rare category (small $n_{k}$ ) has a large contribution to the final distance and moves its owner or owners far away from the others individuals.

While the interpretation of the $\chi^{2}$ distance between individuals is similar to the one given in the $\mathrm{CA}$, the $\chi^{2}$ distance interpretation for variable analysis is quite different and more difficult to justify (Greenacre, 2006). Information contained in a variable can be studied through its categories, thus, MCA focuses mostly on variable categories. As for row-profiles, the distance between categories $k$ and $k^{\prime}$ is defined as the weighted sum of squared distances between the $k$ th and $k^{\prime}$ th column profiles with weights given by the inverse of the average column profile which has in this case all elements equal to $1 / n$ :

$$
d_{k, k^{\prime}}^{2}=n \sum_{i=1}^{n}\left(\frac{x_{i k}}{n_{k}}-\frac{x_{i k^{\prime}}}{n_{k^{\prime}}}\right)^{2}=\frac{1}{p_{k}}+\frac{1}{p_{k^{\prime}}}-\frac{2 p_{k k^{\prime}}}{p_{k} p_{k^{\prime}}}, \quad 1 \leq k, k^{\prime} \leq K,
$$

where $p_{k}=n_{k} / n$ is the relative frequency of the category $k$ and $p_{k k^{\prime}}$ the relative frequency of occurence of categories $k$ and $k^{\prime}$. If $k$ and $k^{\prime}$ are different categories of the same variable, then $p_{k k^{\prime}}=0$. As it is defined, the distance between column profiles is a decreasing function with respect to the relative frequencies $p_{k}$ and joint relative frequencies $p_{k k^{\prime}}$. Two categories are close one to each other with respect to this $\chi^{2}$ distance if they have many individuals in common. Again, rare categories are far away from the others. In brief, it is important to take the frequency of each category into account. However, as remarked by Greenacre (1989) and Greenacre (2006), the terms $1 / p_{k}$ present in the $\chi^{2}$ distance are hard to interpret.

Once that distances between objects (individuals and variables) have been defined, the next step in a MCA is to represent individuals and variables in new orthogonal systems and to make the geometric data analysis on smaller dimension sets (Le Roux and Rouanet, 2004). As in principal component or correspondence analysis, new orthogonal systems are built such that they maximise the projected inertia of the individual cloud or variables on these new orthogonal axis, the inertia being defined as usual as the weighted sum of squared distance of individuals or variables to their barycenter. Each axis represents a certain percentage from the total inertia. However, these percentages in MCA are lower than in CA and more dimensions are needed to interpret properly the analysis. Transition relations link the cloud of individuals with the cloud of categories and a biplot representation is usually used as a joint map of individuals and variable categories. The contribution of each individual to each axis as well as the quality of its representation on each axis are obtained in a similar way to CA. For more details about the graphical representation and all matters connected therewith, see for example Greenacre (2006), Escofier and Pagès (2008, chapter 4), Husson et al. (2016, chapter 3). 


\subsubsection{Log-linear model}

Multivariate categorical data as multidimensional contingency tables (with an order greater than two-way) display relationships between categorical variables. This kind of data can me model by a log-linear model, that is a generalized linear model for Poisson regression. The Poisson distribution is the simplest distribution for count data. The model describes association and interaction among categorical variables and its purpose is to establish dependence patterns between variables. There is no distinction between explanatory or response variables since only the cell counts are considered. The reader may find a comprehensive description in Agresti (2013, chapter 9).

For the sake of simplicity, we present the method for three categorical variables $X_{1}$, $X_{2}$ and $X_{3}$ respectively with $K_{1}, K_{2}$ and $K_{3}$ categories. The most general log-linear model for the three-way table $K_{1} \times K_{2} \times K_{3}$ is written as

$$
\log \mu_{k_{1} k_{2} k_{3}}=\lambda+\lambda_{k_{1}}^{X_{1}}+\lambda_{k_{2}}^{X_{2}}+\lambda_{k_{3}}^{X_{3}}+\lambda_{k_{1} k_{2}}^{X_{1} X_{2}}+\lambda_{k_{1} k_{3}}^{X_{1} X_{3}}+\lambda_{k_{2} k_{3}}^{X_{2} X_{3}}+\lambda_{k_{1} k_{2} k_{3}}^{X_{1} X_{2} X_{3}}
$$

where $\mu_{k_{1} k_{2} k_{3}}$ is the expected frequency of the cell with $X_{1}=k_{1}, X_{2}=k_{2}$ and $X_{3}=k_{3}$. The model-parameters are interpreted as follows: $\lambda$ is the overall effect; $\lambda_{k_{j}}^{X_{j}}$ is the effect of the level $X_{j}=k_{j}, j=1,2,3 ; \lambda_{k_{j} k_{j^{\prime}}}^{X_{j} X_{\prime^{\prime}}}$ is the interaction effect of levels $X_{j}=k_{j}$ and $X_{j^{\prime}}=k_{j^{\prime}}, 1 \leq j, j^{\prime} \leq 3$; finally $\lambda_{k_{1} k_{2} k_{3}}^{X_{1} X_{2} X_{3}}$ is the interaction effect between the levels $X_{1}=k_{1}, X_{2}=k_{2}$ and $X_{3}=k_{3}$. The model (3) is called the saturated model, it includes all possible main effects and interactions between the variables. Some constraints between the parameters ensure model identifiability and the number of free parameters in the saturated model is equal to the number of cells $K_{1} K_{2} K_{3}$, which is why the saturated model fits the data perfectly. It reproduces exactly the observed cell frequencies and does not provide much relevant information.

The aim is to find the simplest model that fits the data adequately, that is, a more parsimonious model with less parameters. An unsaturated model is obtained by imposing the nullity of some coefficients in (3) and may be more appropriate due to simpler interpretations. Validation is performed thanks to goodness-of-fit assessment comparing the expected cell frequencies to the observed frequencies. The goodness-offit can be tested with the likelihood-ratio statistic:

$$
G^{2}=2 \sum_{k_{1}=1}^{K_{1}} \sum_{k_{2}=1}^{K_{2}} \sum_{k_{3}=1}^{K_{3}} n_{k_{1} k_{2} k_{3}} \log \left(\frac{n_{k_{1} k_{2} k_{3}}}{\hat{\mu}_{k_{1} k_{2} k_{3}}}\right),
$$

where $n_{k_{1} k_{2} k_{3}}$ and $\hat{\mu}_{k_{1} k_{2} k_{3}}$ are respectively the cell frequencies and the fitted values from model (3) taking into account the nullity constraint (Agresti, 1990). The $G^{2}$ statistic is used to determine the rejection or acceptance of a model. The larger the value of $G^{2}$, the more evidence there is against that the related model does fit the data adequately, hence it should not be kept.

Different types of unsaturated log-linear models correspond to different type of dependence between the variables $X_{1}, X_{2}$ and $X_{3}$. We will consider here only hierarchical models, meaning that if variables are involved in high order interactions, all the lowerorder interaction term must also appear. For example, if the model contains $\lambda_{k_{1} k_{2}}^{X_{1} X_{2}}$, then it also must contain $\lambda_{k_{1}}^{X_{1}}$ and $\lambda_{k_{2}}^{X_{2}}$. Table 2 summarizes the different types of resulting models which are ordered with increasing complexity. The simplest model, noted $\left(X_{1}, X_{2}, X_{3}\right)$, assumes the nullity of all the interaction effects and corresponds to the mutual independence of $X_{1}, X_{2}$ and $X_{3}$. The model with no interaction of order 3 and 
TAB 2.: Different structures of log-linear models corresponding to different dependence structures. The third column "Symbol" corresponds to model notations, that is, the higher-order model term represented of each variable used in the model.

\begin{tabular}{lll}
\hline Log-linear model & Interpretation & Symbol \\
\hline$\lambda+\lambda_{k_{1}}^{X_{1}}+\lambda_{k_{2}}^{X_{2}}+\lambda_{k_{3}}^{X_{3}}$ & mutual independence & $\left(X_{1}, X_{2}, X_{3}\right)$ \\
$\lambda+\lambda_{k_{1}}^{X_{1}}+\lambda_{k_{2}}^{X_{2}}+\lambda_{k_{3}}^{X_{3}}+\lambda_{k_{2} k_{3}}^{X_{2} X_{3}}$ & independence of $X_{1}$ and $\left(X_{2}, X_{3}\right)$ & $\left(X_{1}, X_{2} X_{3}\right)$ \\
$\lambda+\lambda_{k_{1}}^{X_{1}}+\lambda_{k_{2}}^{X_{2}}+\lambda_{k_{3}}^{X_{3}}+\lambda_{k_{1} k_{3}}^{X_{1}}+\lambda_{k_{2} k_{3}}^{X_{2} X_{3}}$ & independence of $X_{1}$ and $X_{2}$ given $X_{3}$ & $\left(X_{1} X_{3}, X_{2} X_{3}\right)$ \\
$\lambda+\lambda_{k_{1}}^{X_{1}}+\lambda_{k_{2}}^{X_{2}}+\lambda_{k_{3}}^{X_{3}}+\lambda_{k_{1} k_{2}}^{X_{X_{2}}}+\lambda_{k_{1} k_{3}}^{X_{3}}+\lambda_{k_{2} k_{3}}^{X_{2} X_{3}}$ & homogeneous association & $\left(X_{1} X_{2}, X_{2} X_{3}, X_{1} X_{3}\right)$ \\
\hline
\end{tabular}

no interaction of second order between $X_{1}, X_{2}$ and $X_{1}, X_{3}$ is noted $\left(X_{1}, X_{2} X_{3}\right)$ and corresponds to the independence of $X_{1}$ and $\left(X_{2}, X_{3}\right)$. The model with no interaction of order 3 and no interaction of order 2 between $X_{1}$ and $X_{2}$ is noted $\left(X_{1} X_{3}, X_{2} X_{3}\right)$ and corresponds to the conditional independence of $X_{1}$ and $X_{2}$ given $X_{3}$. Finally, the model $\left(X_{1} X_{2}, X_{2} X_{3}, X_{1} X_{3}\right)$ has all interactions of order 2 but no interaction of order 3 and corresponds to homogeneous association that we will explain below. One goal of the analysis of the log-linear model is to find out which is the simplest model suitably fitting the data.

We now discuss marginal and conditional association of variables. A two-way contingency table can be obtained by marginalizing out the third variable, obtaining the so-called marginal table. Associations in this table are summarized by the marginal odd ratios. The marginal odds ratio of a $2 \times 2$ table (of $X_{1}$ and $X_{2}$ ) is defined by

$$
\theta_{X_{1} X_{2}}=\frac{\mu_{11+} \mu_{22+}}{\mu_{12+} \mu_{21+}} .
$$

where $\mu_{i j+}=\sum_{k_{3}} \mu_{i j k_{3}}$ are the expected marginal frequencies with $i, j=1,2$ and $k_{3}$ a fixed category of $X_{3}$.

The distribution of the two variables $X_{1}$ and $X_{2}$ can be displayed conditionally on different levels of $X_{3}$ using cross sections of the three-way contingency table. The associations in these cross-sections (also called partial tables) are called conditional associations and summarized by conditional odds ratios: for instance the ratio of the odds of a $2 \times 2 \times K_{3}$ table is defined by

$$
\theta_{X_{1} X_{2}\left(k_{3}\right)}=\frac{\mu_{11 k_{3}} \mu_{22 k_{3}}}{\mu_{12 k_{3}} \mu_{21 k_{3}}} .
$$

On the other hand, the absence of interaction of order 3 in the model $\left(X_{1} X_{2}, X_{2} X_{3}, X_{1} X_{3}\right)$ implies that the conditional odds ratios do not depend on the category of the third conditioning variable (Agresti, 2013). This property explains the term homogeneous association.

In practice, often data sets contain a large number of categorical variables which may have a large number of levels. Hence, using log-linear models as described before would require a large number of higher order interactions. The estimation and interpretation of parameters may be difficult in such situations. To cope with this difficulty, one can restrict the interaction parameters to have some predefined form, for example a product form as suggested by Andersen (1980), Goodman (1986). The resulting model, known as the multidimensional row-column or the $\mathrm{RC}$ association model is log-multiplicative rather than log-linear since it contains multiplicative terms for the interactions. The number of parameters from log-multiplicative models to be interpreted are considerably reduced in this way. Coefficients used in these multiplicative 
terms are closely related to elements from the singular value decomposition associated to the correspondence analysis of the contingency table as described in Van der Heijden et al. (1989). The simple or multiple correspondence analysis may be also used to detect groups of variables or categories of variables which are mostly related. Then, one can fit a log-linear model using only these groups of selected variables/categories of variables. The resulting log-linear model is no longer hierarchical but the number of interactions is considerably reduced.

\subsubsection{Ordinal regression model}

The logistic regression is a popular supervised learning method for analysing dependencies between a response categorical variable $Y$ (binary or multiclass) and explanatory variables denoted by $\mathbf{X}=\left(X_{1}, \ldots, X_{p}\right)$. More precisely, the logistic regression is used in order to separate the effects of each variable, that is, identify the effects of an explanatory variable $X_{j}, j=1, \ldots, p$, on the response variable $Y$. The logistic regression for a binary or multiclass response variable will be presented briefly below, for more details see for example (McCullagh and Nelder, 1989, chapter 5), (Agresti, 1990, chapter 9) or (Hothorn and Everitt, 2014, chapter 7).

Let $Y \in\{0,1\}$ be a binary response variable. The logistic regression model is written as

$$
\mathbb{P}(Y=1 \mid \mathbf{X}=\mathbf{x})=F\left(\beta_{0}+\boldsymbol{\beta}^{T} \mathbf{x}\right),
$$

where $\mathbf{x} \in \mathbb{R}^{p}, \beta_{0} \in \mathbb{R}, \boldsymbol{\beta} \in \mathbb{R}^{p}$ and $F(t)=e^{t} /\left(1+e^{t}\right), t \in \mathbb{R}$, is the inverse logistic link function. The coefficients $\beta_{0}, \beta_{1}, \ldots, \beta_{p}$ are estimated by maximum likelihood method. Equivalently, the $\log$ odds of the event $\{Y=1\}$ given $\mathbf{X}=\mathbf{x}$ is linear in $\mathbf{x}$ :

$$
\log \operatorname{odds}(Y=1 \mid \mathbf{X}=\mathbf{x})=\log \frac{\mathbb{P}(Y=1 \mid \mathbf{X}=\mathbf{x})}{1-\mathbb{P}(Y=1 \mid \mathbf{X}=\mathbf{x})}=\boldsymbol{\beta}^{T} \mathbf{x} .
$$

Finally, a variable $X_{j}$ reveals to have an effect on the response variable if the result of the nullity coefficient test for $\beta_{j}$ is significant, that means, $\beta_{j}$ not equal to 0 (several nullity tests exist such as Wald test for instance).

Now, in this study, the focus lies on a categorical variable with more than two categories. Let $Y$ be a multiclass response variable. The logistic regression for a multiclass response variable is an extension of the logistic regression for a binary one. When the categories $\left\{m_{1}, \ldots, m_{q}\right\}$ of the response variable $Y$ are hierarchically ordered as $m_{1} \prec \ldots \prec m_{q}$, a way to model $Y$ is to suppose that there exists a latent unobserved continuous variable denoted $Y^{*} \in \mathbb{R}$, with logistic distribution $F$, such that

$$
Y=m_{k} \quad \text { if and only if } \quad c_{k-1}<Y^{*} \leq c_{k},
$$

where $-\infty=c_{0}<c_{1}<\ldots<c_{q-1}<c_{q}=+\infty$ and $k=1,2, \ldots, q$. Then, the ordinal regression model is written as

$$
\mathbb{P}\left(Y \preceq m_{k} \mid \mathbf{X}=\mathbf{x}\right)=F\left(c_{k}-\boldsymbol{\beta}^{T} \mathbf{x}\right),
$$

where $k=1,2, \ldots, q-1$. Note that the general intercept $\beta_{0}$ is replaced by the set of ordered intercept paramaters $c_{k}$ mentioned before. The unknown coefficients $c_{1}, \ldots, c_{q-1}, \beta_{1}, \ldots, \beta_{p}$ are estimated by maximimum likelihood. 
Model (4) is also called the proportional-odds model due to the following property: the $\log$ odds ratio of $\left\{Y \preceq m_{k}\right\}$ at $\mathbf{X}=\mathbf{x}_{\mathbf{1}}$ and $\mathbf{X}=\mathbf{x}_{\mathbf{2}}$ is given by

$$
\log \frac{\operatorname{odds}\left(Y \preceq m_{k} \mid \mathbf{X}=\mathbf{x}_{\mathbf{1}}\right)}{\operatorname{odds}\left(Y \preceq m_{k} \mid \mathbf{X}=\mathbf{x}_{\mathbf{2}}\right)}=-\boldsymbol{\beta}^{T}\left(\mathbf{x}_{\mathbf{1}}-\mathbf{x}_{\mathbf{2}}\right),
$$

and does not depend on the category $m_{k}$.

\section{Results}

This section aims at giving a multivariate analysis of Franche-Comté road crash data by using the above described methods. Our analysis begins by performing several MCA analyses on road crash variables to provide insights multivariate road crash related variables by using geometric data visualization. This method is a powerful tool to distinct non-trivial category associations if it is the case. More exactly, MCA analyzes were performed by considering temporal and spatial features separately and next, a global MCA analysis based on temporal, spatial features and variables which revealed to be most related one to another from separate MCA analysis. General features such as crash gravity, alcohol/drug consumption, road type and so on were included in the three performed MCA analyzes. These MCA analyzes revealed several association and interaction patterns among the set of categorical variables related to road crash gravity and hierarchical and non-hierarchical log-linear models were fitted in second time to describe more thoroughly these associations. Finally, the ordinal regression model allows to quantify the effects of each explanatory variable on the ordered response variable road crash gravity.

This study used open-source R software packages FactoMineR (Lê et al., 2008) and factoextra (Kassambara and Mundt, 2019) to perform MCA, glm function to perform log-linear models, then packages MASS (Venables and Ripley, 2002) and ordinal (Christensen, 2019) to perform ordinal logistic regression. Graphics were plotted with ggplot2 package (Wickham, 2016).

\subsection{MCA of road crash temporal variables}

We conducted a MCA temporal analysis by considering general and temporal features as described in Section (2.1). Spatial features are not included in MCA analysis. FIG (2) gives the percentages of variance explained by each of the first ten axes built by the MCA analysis. The first three-factorial axes explain $22,09 \%$ of the total variance and only these axes were kept for further analysis. Two-dimensional geometrical representations are given in FIG (3)-(5) and interpreted below. Each time, only the 25 best represented categories have been plotted.

The two-dimensional map in FIG (3) gives the representation of categories on the plane made by axis 1 and axis 2 and it accounts for $16,07 \%$ of the total inertia. The more categories a variable has, the more it contributes to the inertia. The variables season and weather are not represented on the first factorial plane since they are very poorly represented on this plane. Next, categories with the greatest contribution to the axis 1 are "night" (13,26\%), "none" from obstacle (10,57\%) and "midnight_6am" $(9,26 \%)$ and respectively, "pedestrian" $(28,45 \%)$, "urban" $(19,04 \%)$ and "other_kind" from collision $(11,46 \%)$ for axis 2 . 
In this first factorial plane, axis 1 shows the contrast between weekday accidents (accidents occurring during the week) and weekend ones (accidents occurring during the weekend). Weekday accidents are more frequent during the day and mostly around lunch time, in urban areas, on communal roads and are not associated to alcohol or drug consumption. These accidents are more likely to happen on straight roads, at intersections and caused by collisions between several vehicles. Weekend accidents, instead, are more frequent during the night and mostly between $8 \mathrm{pm}$ and $6 \mathrm{am}$, outside urban areas and involve more frequently drug consumers. These accidents occur mainly on curve roads and no external factors seem to impact (out of intersections, no bumped mobile obstacles or no collisions). To sum up, this first factorial axis is related to fatal accidents.

Axis 2 provides a similar information as axis 1: it opposes accidents occurred during weekday time, in urban area, at intersection and on straight road to accidents occurred during weekend time, outside urban area, out of intersection and on curve road. However, axis 2 stresses the fact that fatal road crash are more likely to occur between midnight and 6 am especially when alcohol and drugs have been consumed.

Geometrical representations derived in MCA plot closely associated categories and unassociated ones further apart. The first factorial plane reveals several strong associations among categories: categories "straight", "intersection", "weekday", "substance_none", "11am_3pm", "collision_usual" are strongly associated to categories "obstacle_vehicle"; categories "curve", "out_of_intersection, "week_end", "unurban" are strongly associated to "fatal"; "night", "8pm_11pm", "midnight_6am", "obstacle_none", "alcohol" and "alcohol_drug" in the same way.

On the other hand, this factorial plane also shows that some categories are far from the others, this results from their lower frequencies. Indeed, as it is given in TAB 1, "obstacle_pedestrian" and "type_road_other_kind" represents respectively only 8,28\% and $2,53 \%$ of road accidents.

FIG (4) gives the two-dimensional map of axis 1 and axis 3 and it explains 15,32\% of the total inertia. The variable area has been omitted from this geometrical representation due to its poor representation quality. Categories that contribute the most to axis 3 are categories "night" $(11,82 \%)$, "none" $(11,08 \%)$ of the variable collision and category "winter" $(10,96 \%)$ of the variable season.

In this factorial plane, axis 3 suggests that summer accidents tend to differentiate from winter ones. Summer accidents are more likely to occur during the day, around lunch time and on week-end time. They are globally associated to no alcohol or drug consumption, happening on curve roads, out of intersections and other kind of collision. Winter accidents instead occur more frequently during the night, the week time and on national roads. They are also mostly associated with substances consumed, happening on straight roads, at intersections and collisions with vehicles. The associations with straight roads, intersections and collisions with vehicles seem to be caused by weather ("other_kind") which is generally snowy in winter. In addition, the axis 3 specifies that winter accidents are more likely to be fatal.

Two groups of strongly associated categories stand out in the second factorial plane: the group formed by "obstacle_vehicle", "collision_usual", "intersection", "straight", "weekday", "substance_none", "day", "11am_3pm" and the other group formed by "summer"; "night", "8pm_11pm", "midnight_6am", "alcohol_drug". These groups resonates to those mentioned previously.

Fig (5) gives the two-dimensional map of axis 2 and axis 3 and it explains 12,79\% of the total inertia. From the thirteen variables used, the variables substance, week and intersection are very poorly represented on this map and are omitted from this ge- 


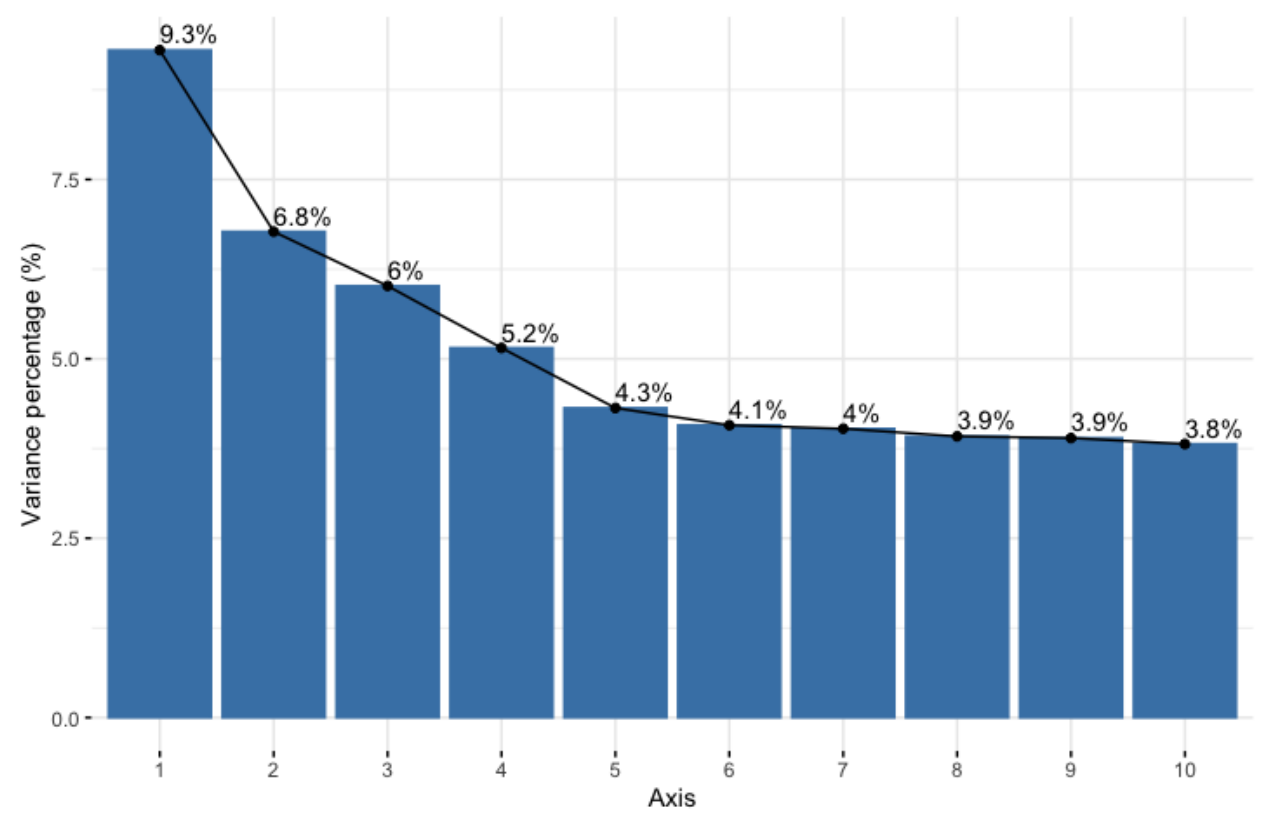

FIG 2.: MCA temporal analysis: variance percentage explained by the first 10 axes.

ometrical representation. This plot emphasizes the differences between serious and fatal accidents which tend to be strongly associated with lunch time and respectively night time. We distinguish two groups of close categories: "winter", "night", "fatal", "8pm_11pm", "obstacle_vehicle", "collision_usual" and "weather_other_kind"; "type_road_departmental", "serious", "weather_normal", "spring", "curve", "day", "summer", "11am_3pm" and "obstacle_none". 


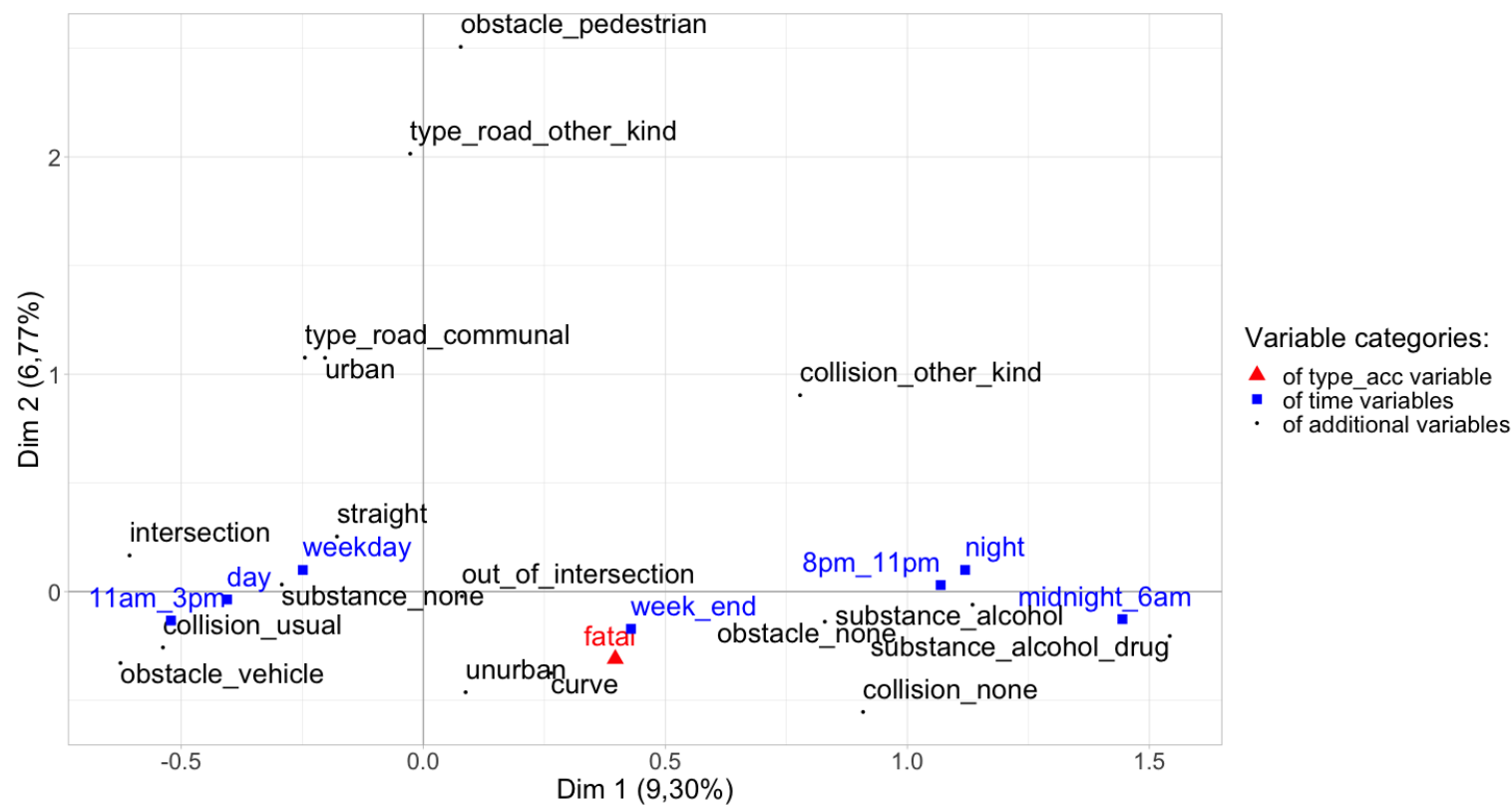

FIG 3.: MCA temporal analysis: factorial plane made by axis 1 and axis 2 .

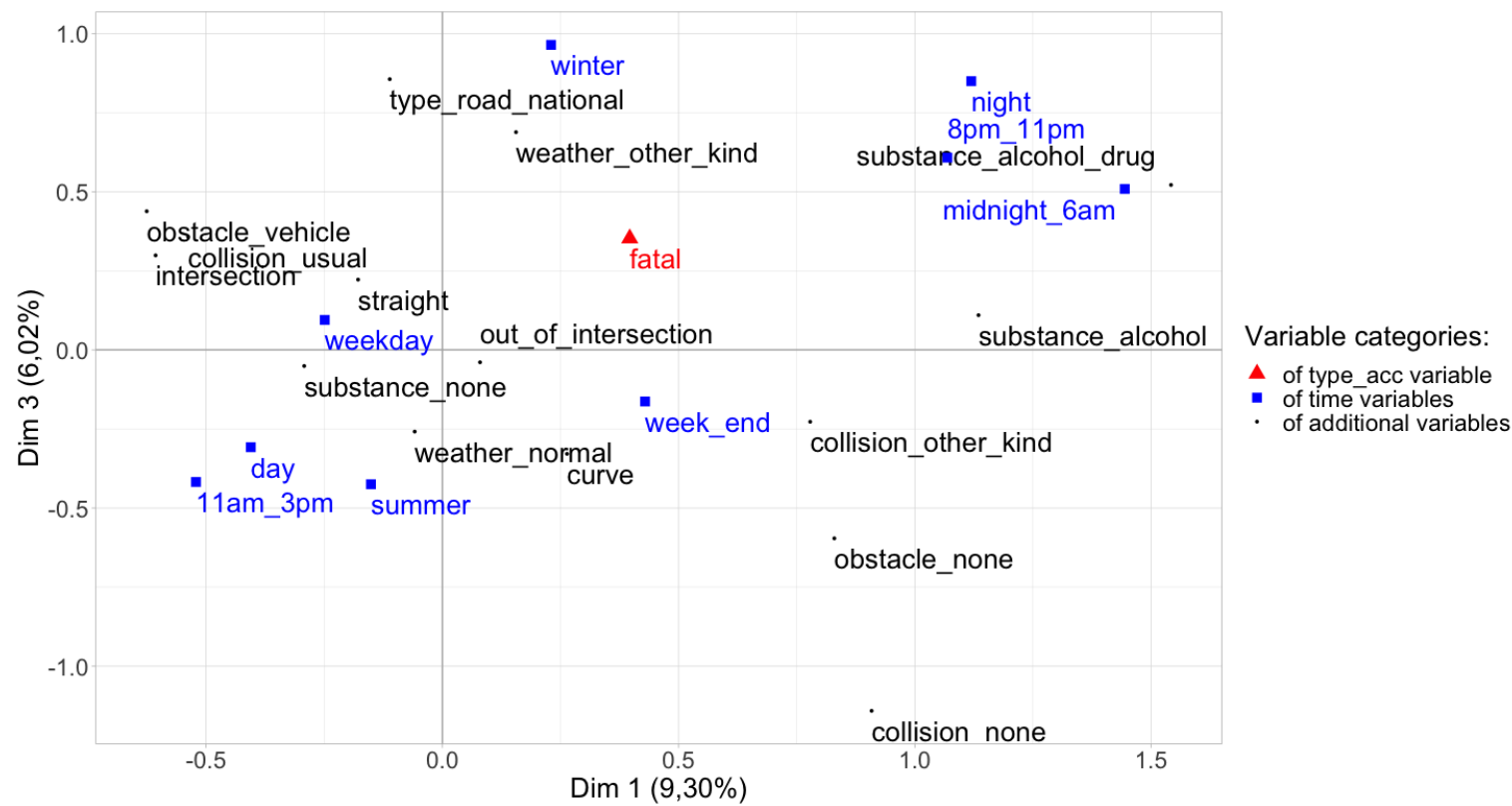

FIG 4.: MCA temporal analysis: factorial plane made by axis 1 and axis 3 . 


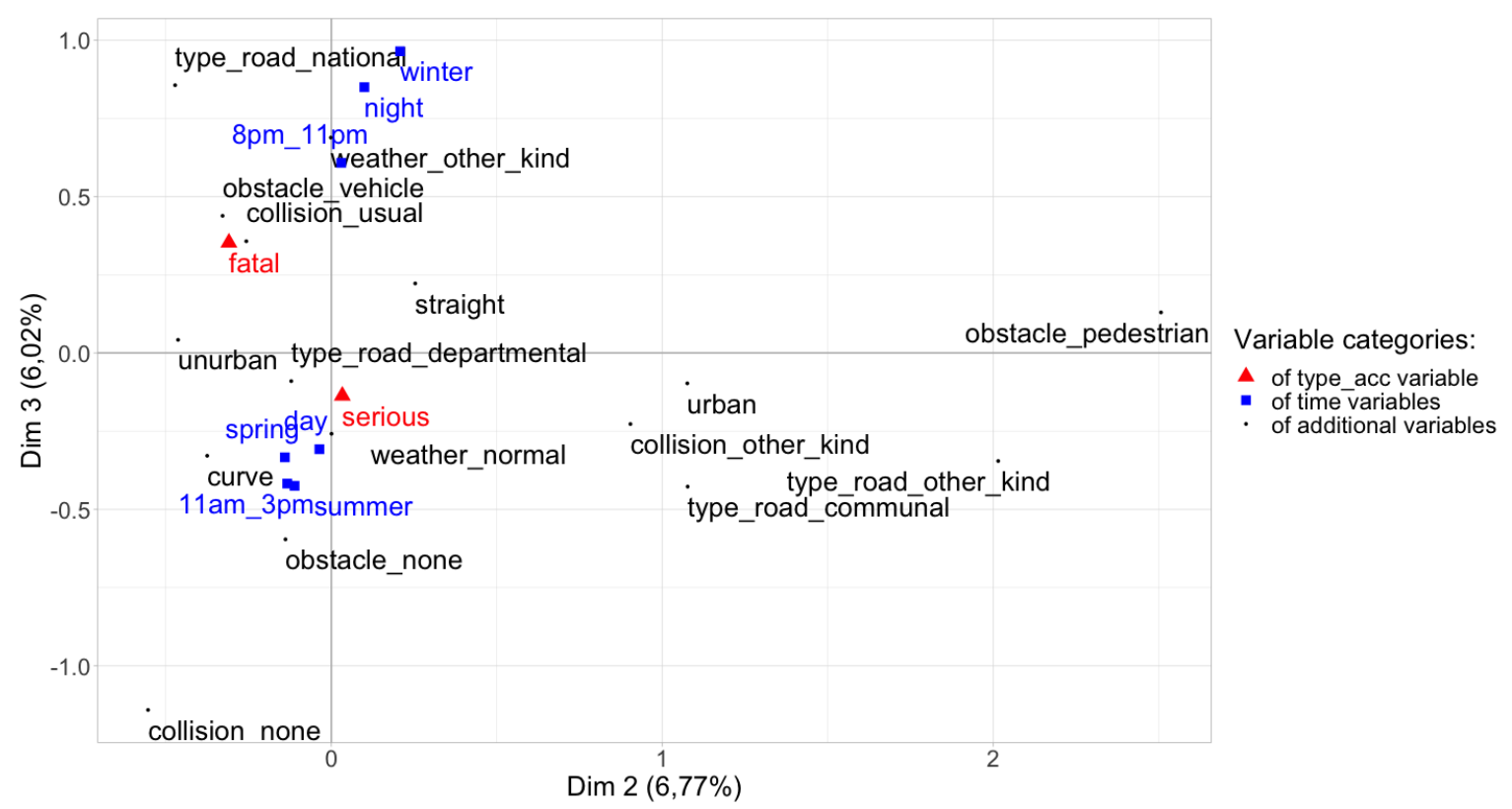

FIG 5.: MCA temporal analysis: factorial plane made by axis 2 and axis 3 .

\subsection{MCA of road crash spatial variables}

We performed a spatial analysis by considering the general features as well as the spatial ones as described in the Section (2.1). Temporal variables (season, week, daytime and time) are not considered in this analysis. Due to the several categories of the variable canton, the spatial analysis of Franche-Comté has been splitted into two analyses corresponding to Doubs and Jura departments. We will interpret only relationships from categories situated close one to another in the plot.

\subsubsection{Doubs department}

For the spatial analysis of Doubs department, the first four factorial axes explain $21,22 \%$ of the total inertia. Note that only the first 25 categories with the most important representation qualities were plotted.

Plot made by axis 1 and axis 2 given in FIG (7) explains 12,74\% of the total inertia. Not all the variables are plotted, the variable weather is less well represented than the other categories and it does not figure on the plot. Categories which contribute the most for axis 1 are "none" $(20,77 \%)$ and "vehicle" $(14,06 \%)$ from obstacle, then "usual" from collision (12,88\%); and for axis 2"pedestrian" (22,53\%), "urban" (18,92\%) and "other_kind" from collision $(9,73 \%)$.

As mentioned before, associations can be highlighted by the proximity of categories on the factorial plot. Two groups of close categories with spatial connotations stand out: "type_road_other_kind", "type_road_communal", "Bethoncourt", "urban" and "Valentigney"; "Besançon", "collision_usual" and "obstacle_vehicle". The first group emphasizes that Bethoncourt and Valentigney accidents are more frequent in urban areas. The second one strongly insists that the canton of Besançon is more conducive to collisions with vehicles.

Additional plots made by combinations of axis 1, 2, 3 and 4 explain between 8,48\% and $11,01 \%$ of the total inertia, it should be noted that compared to each other the as- 


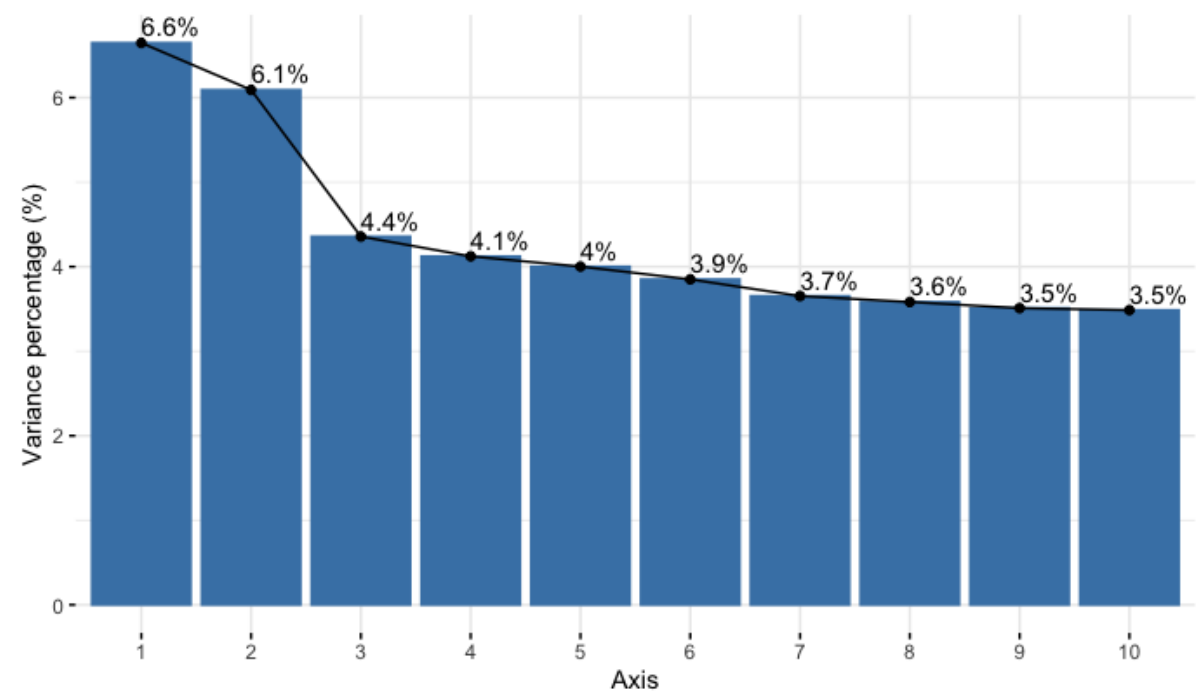

FIG 6.: MCA spatial analysis, Doubs department: variance percentage on the first 10 axes.

sociations do not differ. These plots indicates, in addition to what was said before, that Besançon accidents tend to be fatal when the weather is bad and more characterized by bumped pedestrians; Bethoncourt accidents are mostly associated with substance consumption and very strongly associated to "drug"; Baume-les-Dames accidents are more likely to be fatal; Besançon, Saint-Vit and Ornans accidents are mainly similar and more frequent on communal and national roads; most of Maîche accidents are not caused by collisions.

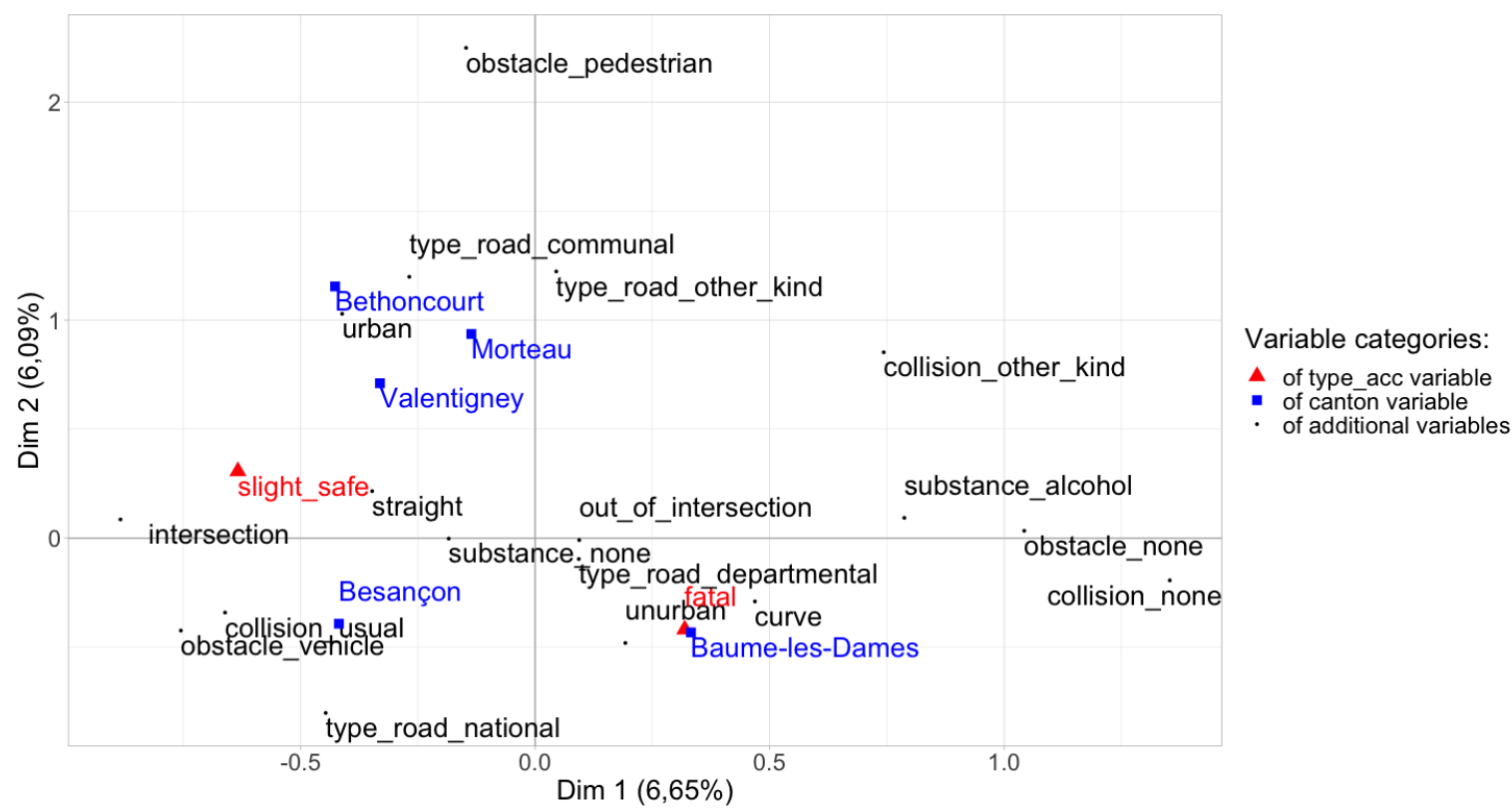

FIG 7.: MCA spatial analysis, Doubs department: factorial plane made by axis 1 and axis 2 . 


\subsubsection{Jura department}

For the spatial analysis of Jura department, the first four factorial axes explain $21,88 \%$ of the total inertia. Note that only the first 25 categories with the most important representation qualities were plotted.

Plot made by axis 1 and axis 2 explains 13,08\% of the total inertia and is given in FIG (9). Not all the variables are plotted, the variable weather is less well represented than the other categories and it does not figure on the plot. Categories which contribute the most for axis 1 are "none" (18,34\%) and "vehicle" (13,79\%) from obstacle, then "usual" from collision $(11,94 \%)$; and for axis 2 "pedestrian" $(24,89 \%)$, "urban" $(18,69 \%)$ and "other_kind" from type_road (11,23\%).

We can distinguish three groups of close categories, with components of the canton variable that stand out in this factorial plane: "Saint-Lupicin", "fatal", "drug", "alcohol_drug", "alcohol" and "obstacle_none"; "Authume", "Saint-Laurenten-Grandvaux", "unurban", "type_road_departmental" and "out_of_intersection"; "Champagnole", "urban" and "type_road_communal". The first group highlights that accidents in the canton of Saint-Lupicin are more likely to be fatal and associated to alcohol and drug consumption. The second one tells that accidents happening in Authume and Saint-Laurent-en-Grandvaux are more frequent in non urban areas, on departmental roads. Then, the third group of close categories shows that accidents in the canton of Champagnole are occuring more commonly in urban areas and on communal roads. Finally, the structure of this factorial plane tells that accidents happening in Authume, Saint-Laurent-en-Grandvaux and Saint-Lupicin are more likely to be fatal.

Additional plots made by combinations of axis 1, 2, 3 and 4 explain between 8,81\% and $11,24 \%$ of the total inertia, it should be noted that compared to each other the associations do not differ. The cantons of Champagnole, Morez and Saint-Laurent-enGrandvaux have been associated to each other in many factorial planes, it seems that accidents are more likely to happen in these cantons when the weather is qualified as "other_kind" (cloudy, rainy or snowy). This is opposed to accidents happening in Authume and Bletterans where accidents are more frequent when the weather is "normal". Many cantons have been related to alcohol or drug consumption: accidents occurring in the Dole canton are more commonly associated to drug, Moirans-en-Montagne and Saint-Claude cantons are instead matched to alcohol. Finally, the canton where accidents happen in higher proportion on highway is Dole, it is also the canton where accidents are more likely to be fatal, and finally, the canton where pedestrians are bumped in much higher amounts is Champagnole.

\subsection{MCA of road crash temporal and spatial variables}

Here was conducted a global MCA by considering both temporal variables (time, season, daytime and week) and spatial variables (department) as well as the most important general features such as type_acc and substance. The first three-factorial axes explain $25,85 \%$ of the total variance and only these axes were kept for further analysis. Two-dimensional geometrical representations of all 24 categories are given in FIG (10)-(12) and interpreted below.

The first two-dimensional map in FIG (10) gives the representation of categories on the plane made by axis 1 and axis 2 and it accounts for $19,13 \%$ of the total inertia. Categories with the greatest contribution to the axis 1 are "night" $(25,75 \%)$, "midnight_6am" (13,83\%) and "8pm_11pm" (11,88\%) and respectively, "winter" (21,99\%), 


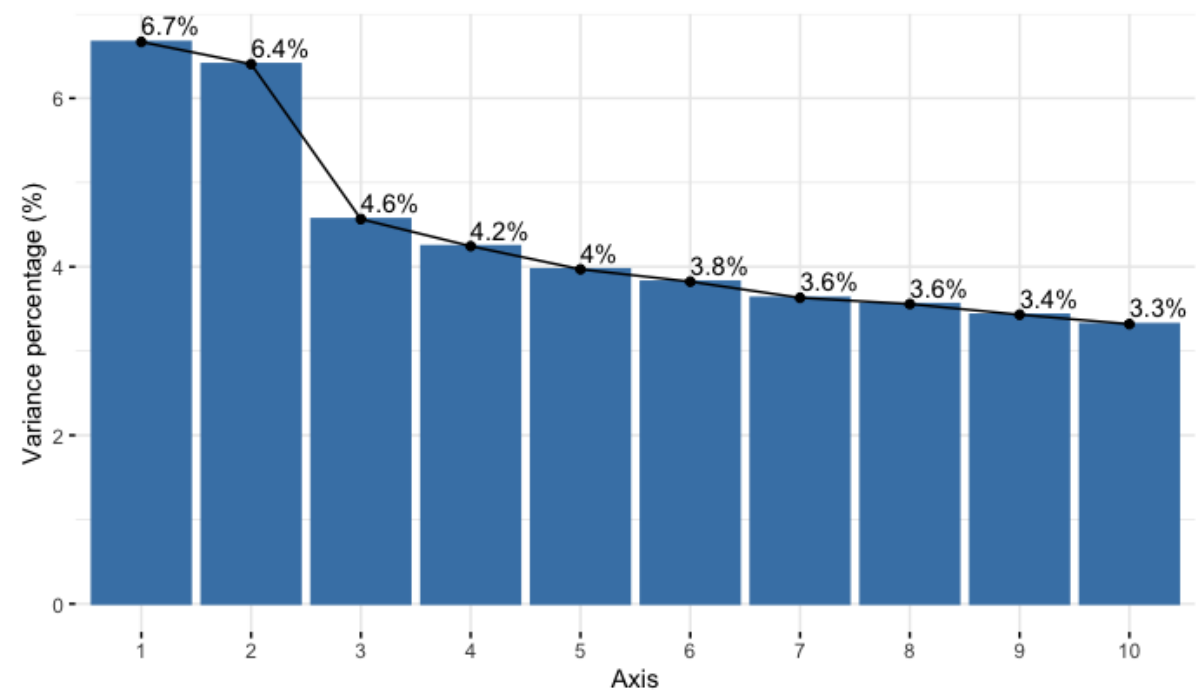

FIG 8.: MCA spatial analysis, Jura department: variance percentage on the first 10 axes.

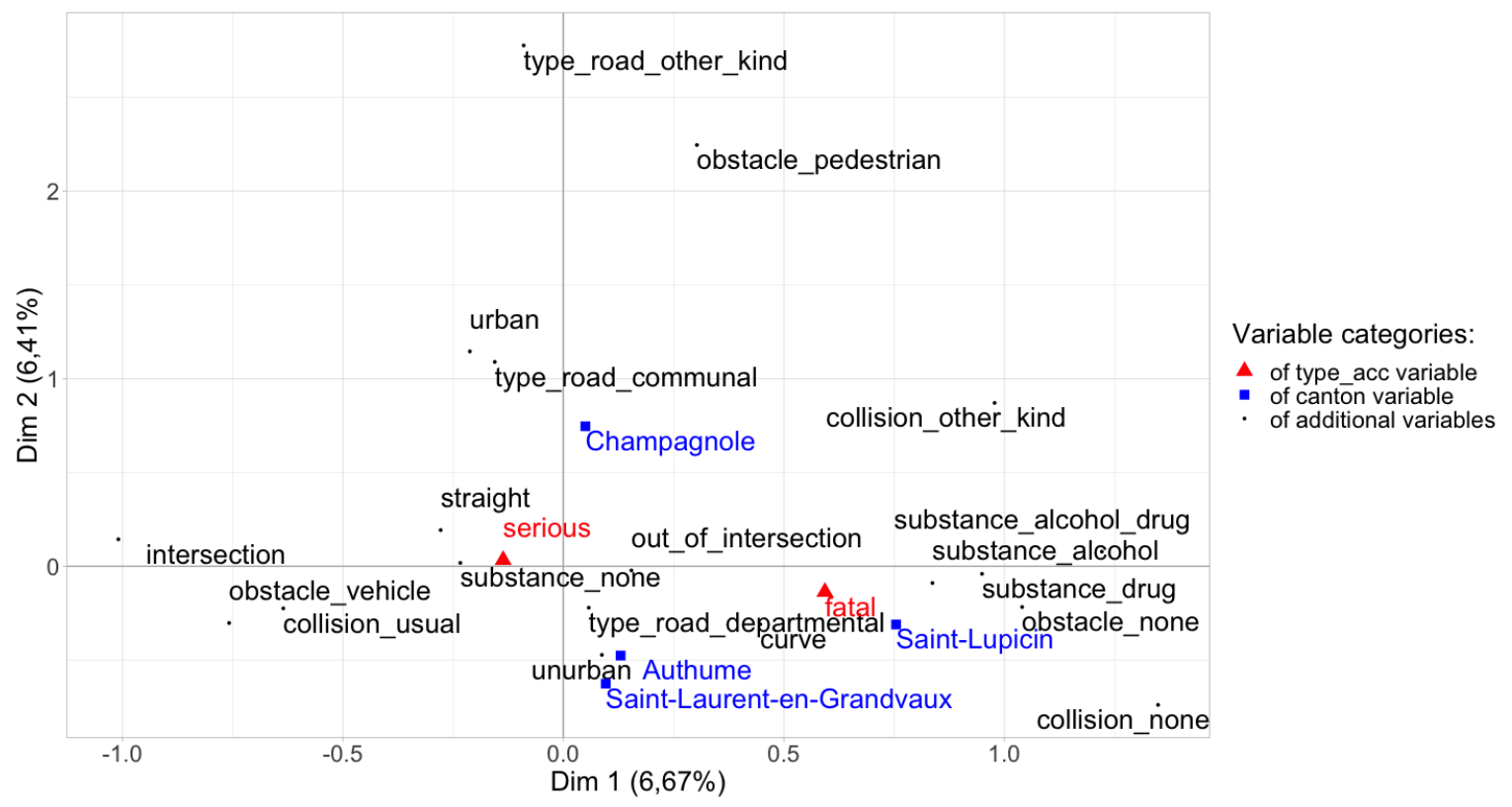

FIG 9.: MCA spatial analysis, Jura departement: factorial plane made by axis 1 and axis 2 .

"slight_safe" $(21,10 \%)$ and "week_end" $(9,33 \%)$ for axis 2 .

Axis 1 shows the contrast between serious accidents occurring during the day (between 7:00 a.m. and 7:59 p.m.) from fatal accidents happening during the night (between 8:00 p.m. and 6:00 a.m.). In the first situation, the accidents happen under good daylight conditions, during the spring and the summer time and mostly during the week-time (from Monday to Friday). In the second situation, accidents occur during the week-end and they are associated to alcohol/drug consumptions.

Axis 2 dissociates clearly slight accidents from serious and fatal ones. Accidents 
occurring in Territoire de Belfort are mostly slight accidents while accidents from Jura are in general serious or fatal. As for the first factorial axis, axis 2 shows the contrast week accidents from week-end accidents which are fatal most of the time. We can notice that accidents occurring in the Doubs department are similar to those from the department of Haute Saône and relatively close to the origine represented by the average accident profile.

The first factorial plane reveals five groups of associated categories: "slight_safe", "Terr_Belfort" and "winter"; "weekday", "7am_10am" and "none"; "fatal", "week_end" and "drug"; "serious", "spring", "summer" and "Jura"; enfin "night", "8pm_11pm", "alcohol_drug", "midnight_6am" and "alcohol".

Notice also that from this plot, the group formed by categories "Territoire Belfort", "winter" and "slight_safe" is relatively far away from the origin of the plot. This is due to the fact that categories "Territoire Belfort" and "slight_safe" are of low relative frequencies, $6,20 \%$ and respectively $11,47 \%$, see also TAB 1 .

Plot made by axis 1 and axis 3 given in FIG (11) explains 18, $87 \%$ of the total inertia. Categories with the greatest contribution to the axis 3 are "Terr_Belfort" (14,96\%), "slight_safe" $(12,84 \%)$ and "spring" $(10,87 \%)$.

Axis 3 seems to contrast accidents occurring in spring/summer from those occurring in autumn/winter. In the first situation, accidents happen mostly during the day, on week-end and they may have minor consequences or, on the contrary, they may be very serious. In the second situation, the accidents occur during the night, on week-time and they may be serious. Again, the department of Jura is in opposite situation with the department Territoire de Belfort. We can also notice that the department Doubs seems to be the department where most of accidents are associated to drugs. Several groups of categories can be distinguished: "drug", "Doubs" and "summer"; "Haute_Saone" and "serious"; "none", "4pm_7pm" and "weekday"; "Jura", "autumn" and "winter".

Finally, the two-dimensional map of axis 2 and 3 given in FIG (12) explains 13,70\% of the total inertia. This plot highlights several associations that were analyzed in previous ones. For example, fairly similar modalities are "8pm_11pm" and "Doubs"; "spring", "11am_3pm", "drug", "week_end", "summer" and "fatal". 


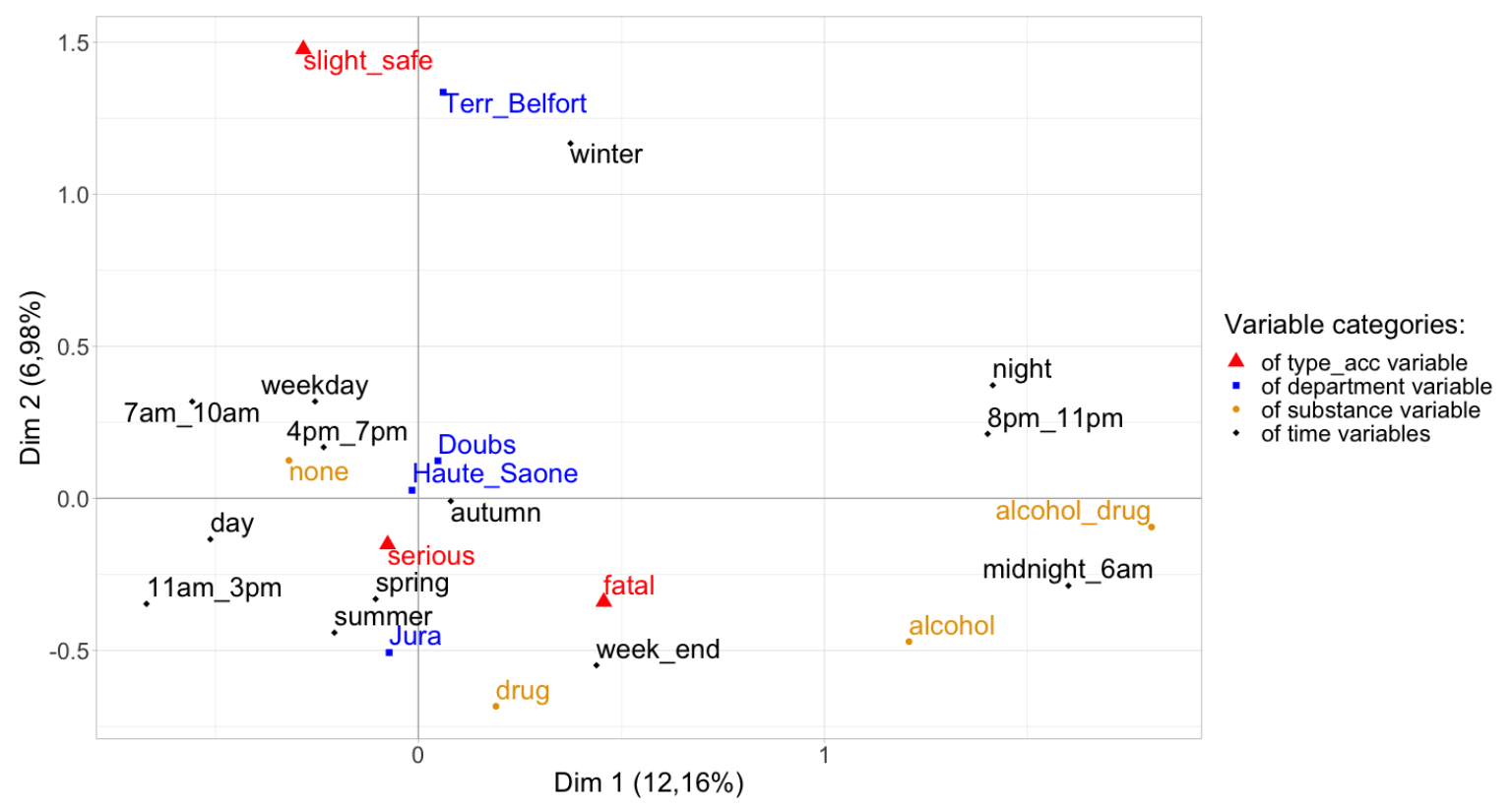

FIG 10.: MCA global analysis: factorial plane made by axis 1 and axis 2 .

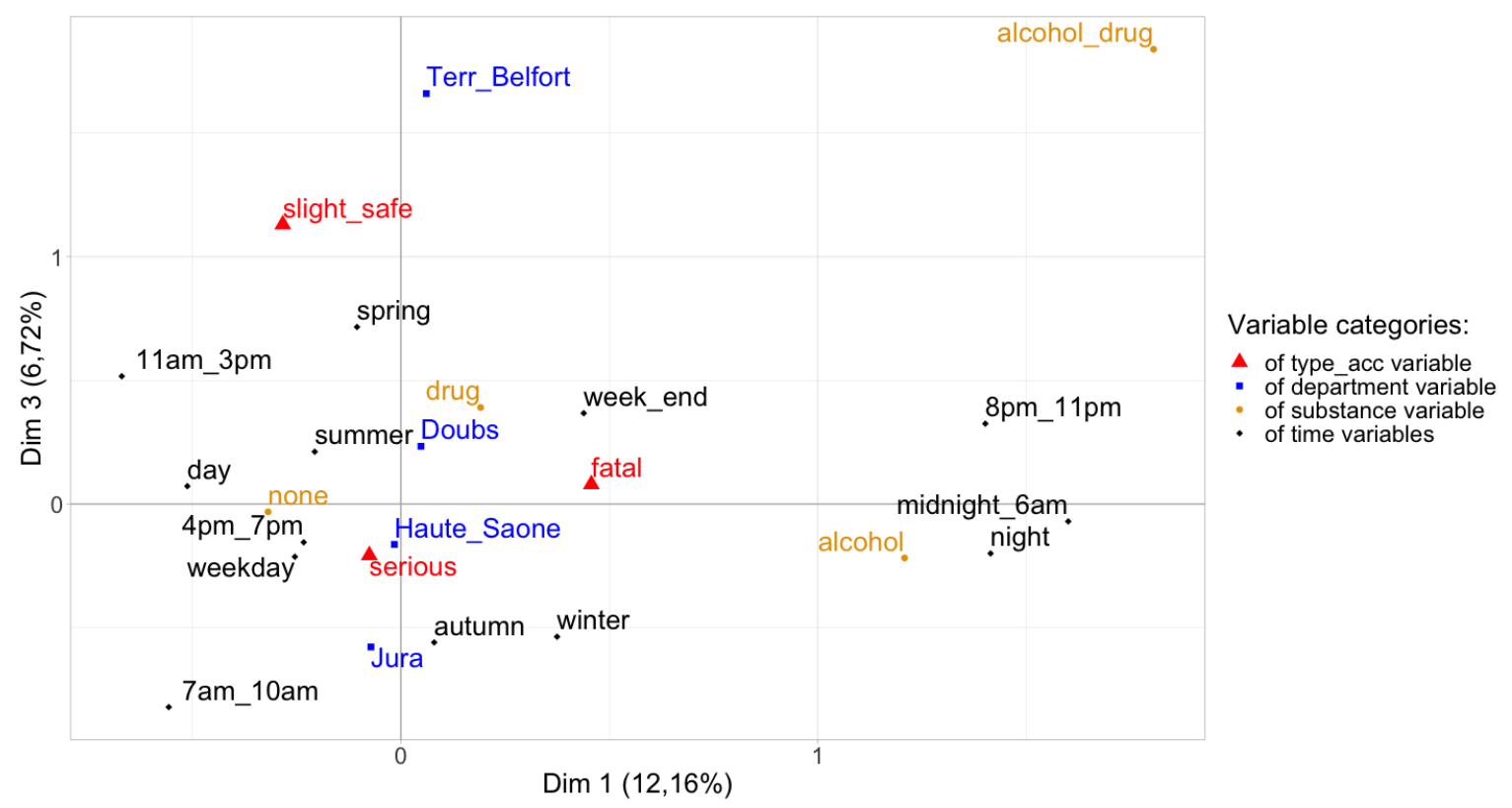

FIG 11.: MCA global analysis: factorial plane made by axis 1 and axis 3 .

\subsection{Hierarchical log-linear modelling}

The MCA performed in sections 3.1 and 3.2 reveals that there are associations between the gravity of the accidents (type_acc) and the drug/alcohol consumption (substance). Moreover, we could see during the spatial analysis that these associations are observed within each department. In order to describe more thoroughly the association patterns between these categorical variables and the variable department, several hierarchical log-linear models have been fitted on the related three-way contingency table (corresponding to the column "Observed values" of the table TAB 3). 


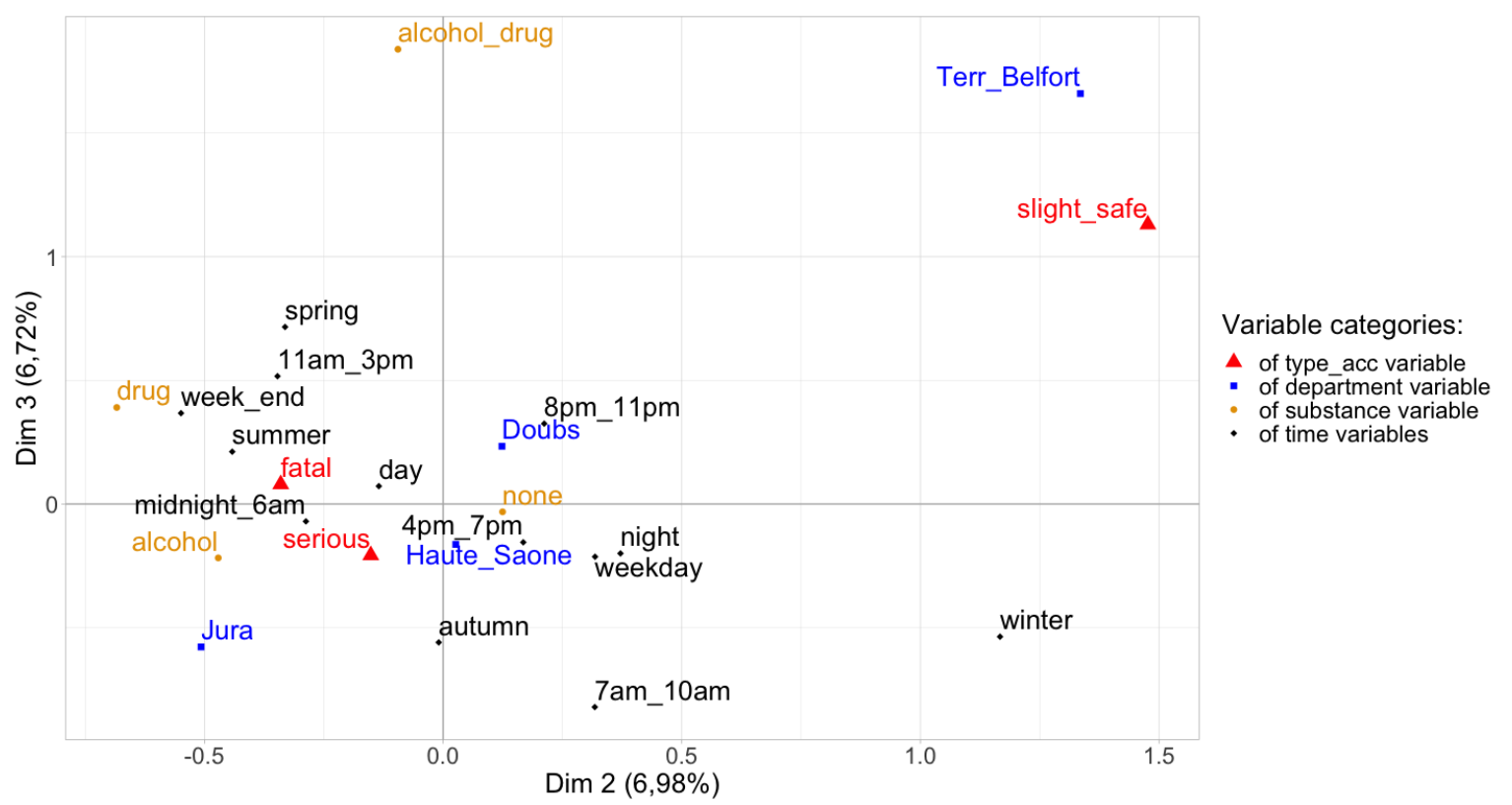

FIG 12.: MCA global analysis: factorial plane made by axis 2 and axis 3 .

TAB 4 gives the likelihood-ratio statistic $G^{2}$ of each hierarchical model and the $5 \%$-level associated significance test (p-value). A model fits the data well if the null hypothesis of the goodness-of-fit test is accepted. The p-values in TAB 4 show that all the models fit the data poorly except (TS, TD, SD) which is close to the observed data (corresponding to the column "Fitted values" of the table TAB 3). This unsaturated hierarchical final model has been kept as the objective was to find the simplest model that fits the data adequately. It is written as

$$
\log \mu_{k k^{\prime} k^{\prime \prime}}=\lambda+\lambda_{k}^{T}+\lambda_{k^{\prime}}^{S}+\lambda_{k^{\prime \prime}}^{D}+\lambda_{k k^{\prime}}^{T S}+\lambda_{k k^{\prime \prime}}^{T D}+\lambda_{k^{\prime} k^{\prime \prime}}^{S D},
$$

where $k$ is "slight_safe", "serious" or "fatal" for the categorical variable type_acc (T) ; $k^{\prime}$ is "none", "alcohol", "drug" or "alcohol_drug" for the variable substance (S) ; and $k^{\prime \prime}$ is "Doubs", "Haute_Saone", "Jura" or "Terr_Belfort" for the variable department (D).

This is the model with no three-factor interaction. The conditional association terms appear for each pair of variables, this means that no pair is conditionally independent. The odds ratios related to this model have been calculated and are given in TAB 5 . Note that the baseline categories of type_acc, substance and department were respectively "slight_safe", "none" and "Doubs". For instance, the odds ratio relating the level "serious" of type_acc and "alcohol_drug" of substance at the level "Doubs" of department is calculated as

$$
\frac{25,64 \times 221,37}{1149,58 \times 3,96}=1,25 .
$$

Remind that the no three-factor interaction model means that the association between two variables is identical at each level of the third variable. Hence, in the same way, calculating this odds ratio with the fitted values regarding to the levels "Haute_Saone", "Jura" or "Terr_Belfort" of department would have given the same result. 
In general marginal odds ratios may differ from conditional ones in a no threefactor interaction model, however in this case marginal and conditional odds ratio are very close. This means that controlling or ignoring the third variable does not change significantly the association between the two variables. Only conditional odds ratio will be interpreted below as the interpretations of marginal ones are the same.

Regarding substances consumption, the odds for an accident to be serious or fatal increases when alcohol, drug or both are consumed. Indeed, the odds ratios for an accident to be serious are estimated to be respectively 1,64, 1,94 and 1,25 higher than slight when alcohol, drug and both are consumed compared with no consumption. Similarly, the odds ratios for an accident to be fatal are estimated to be respectively 2,76, 4,15 and 5,93 higher than slight when alcohol, drug and both are consumed. The highest risk for an accident to be fatal corresponds to drug and alcohol consumption, almost two times larger than alcohol consumption. Regarding the department where the accident happens, the odds to be serious or fatal decreases only for the department Territoire de Belfort. The odds ratio for an accident to be serious is estimated to be 0,48 times lower than slight when it occurs in this department compared with Doubs. Similarly, the odds for an accident to be fatal is estimated to be 0,39 times lower than slight when it occurs in Territoire de Belfort compared with Doubs. The highest risk for an accident to be fatal is when it occurs in Jura department compared with Doubs, almost four times larger than in Territoire de Belfort.

TAB 3.: Three-way contingency table with type_acc, substance and department as categorical variables. Left side correspond to the observed values, right one is equal to the predicted frequencies from the log-linear model (TS, TD, SD).

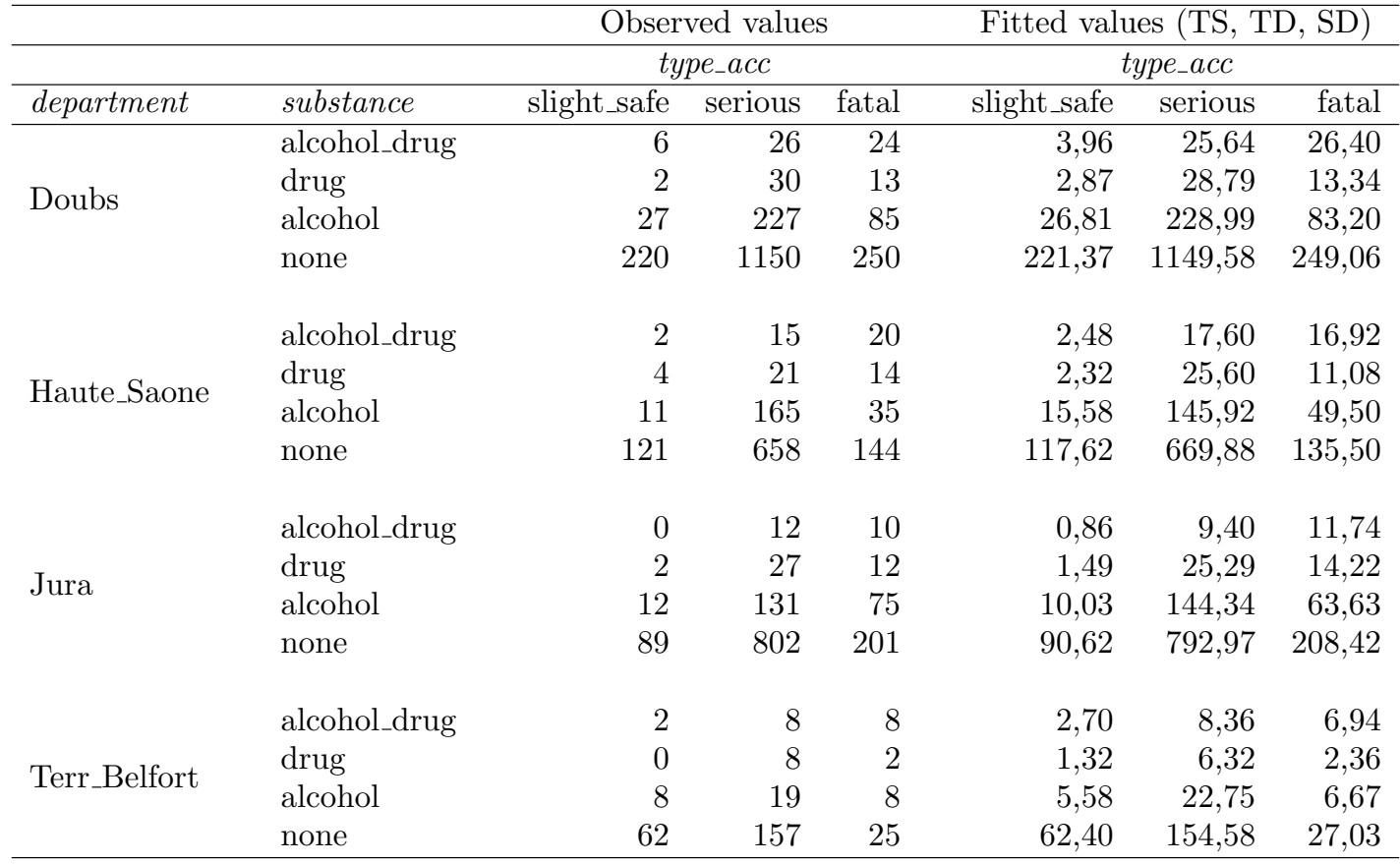


TAB 4.: Goodness-of-Fit Tests for log-linear models relating type_acc (T), substance (S) and department (D).

\begin{tabular}{lrr}
\hline Model & \multicolumn{1}{c}{$G^{2}$} & p-value \\
\hline (T, S, D) & 246,39 & 0,00 \\
& & \\
(T, SD) & 218,97 & 0,00 \\
(S, TD) & 179,65 & 0,00 \\
(D, TS) & 125,36 & $1,09 \mathrm{e}-12$ \\
& & \\
(TS, TD) & 58,61 & $3,99 \mathrm{e}-4$ \\
(TS, SD) & 97,94 & $6,72 \mathrm{e}-11$ \\
(TD, SD) & 152,23 & 0,00 \\
& & \\
(TS, TD, SD) & 27,38 & 0,07 \\
& & - \\
(TSD) & 0,00 & \\
\hline
\end{tabular}

TAB 5.: Odds ratio estimated from (TS, TD, SD) log-linear model. The table is divided into two parts, which are also splitted up into two parts: conditional odds ratios in top have been calculated respectively in left and right sides controlling levels of department and substance variable, and marginal odds ratios in bottom have been calculated respectively in left and right sides ignoring department and substance variable. Each odds ratio has been calculated as each level of type_acc, substance and department variable and was opposed respectively to "slight_safe", "none" and "Doubs".

\begin{tabular}{lrrlrr}
\hline \multicolumn{7}{c}{ Conditional odds ratios } \\
\hline alcohol_drug & serious & fatal & serious & fatal \\
drug & 1,25 & 5,93 & Haute_Saone & 1,10 & 1,02 \\
alcohol & 1,94 & 4,15 & Jura & 1,69 & 2,05 \\
\hline \multicolumn{7}{c}{ Marginal odds ratios } & 0,48 & 0,39 \\
\hline & 1,64 & 2,76 & Terr_Belfort & serious & fatal \\
alcohol_drug & serious & fatal & & 1,11 & 1,06 \\
drug & 1,08 & 4,92 & Haute_Saone & 1,68 & 1,98 \\
alcohol & 1,91 & 4,07 & Jura & 0,47 & 0,41 \\
\hline
\end{tabular}

\subsection{Log-linear modelling using $M C A$}

The goal here was to fit a log-linear model with more than three categorical variables. In order to do that, the global MCA analysis performed in the previous section has been used to choose several variables associated to the road crash severity and to fit a log-linear model with a limited number of parameters but well chosen. A similar analysis has been performed by Papagiotakos and Pitsavos (2004). The global MCA analysis revealed several groups of associated variables/categories such as "slight_safe", "Terr_Belfort" and "winter"; "night", "8pm_11pm", "alcohol_drug", "midnight_6am" and "alcohol", or even more "fatal", "week_end" and "drug". These three groups sug- 
gest that it might exist interactions between the variables type_acc, department and season ; daytime, time and substance ; and finally, type_acc, week and substance.

Based on these considerations, the following log-linear model has been fitted:

$$
\log \mu_{k_{1} k_{2} k_{3} k_{4} k_{5} k_{6} k_{7}}=\lambda+\lambda_{k_{1} k_{3}}^{T D}+\lambda_{k_{2} k_{3}}^{S D}+\lambda_{k_{7} k_{4} k_{2}}^{D a T i S}+\lambda_{k_{1} k_{6} k_{2}}^{T W S}+\lambda_{k_{1} k_{3} k_{5}}^{T D S e}+\lambda_{k_{6} k_{4} k_{2}}^{W T i S}+\lambda_{k_{4} k_{2} k_{3}}^{T i S D},
$$

where $k_{1}$ is "slight_safe", "serious" or "fatal" for the categorical variable type_acc (T); $k_{2}$ is "none", "alcohol", "drug" or "alcohol_drug" for the variable substance $(\mathrm{S}) ; k_{3}$ is "Doubs", "Haute_Saone", "Jura" or "Terr_Belfort" for the variable department (D); $k_{4}$ is "7am_10am", "11am_3pm", "4pm_7pm", "8pm_11pm" or "midnight_6am" for the categorical variable time (Ti); $k_{5}$ is "spring", "summer", "autumn" or "winter" for the variable season (Se); $k_{6}$ is "weekday" or "week_end" for the variable week (W); and $k_{7}$ is "day" or "night" for the variable daytime (Da).

Several different log-linear models have been fitted and compared one to each other, this final model was the one with the smallest likelihood-ratio statistic $G^{2}$. This loglinear model contains specific second and third order interactions between the seven chosen variables based on the global MCA. As mentioned before, these interactions have been decided upon the groups of associated variables revealed in the global MCA. Considering all the second and third interaction terms in the log-linear model would cost in term of interpretation and feasibility.

TAB 6.: Seven-way contingency table with type_acc (T), department (D), substance $(\mathrm{S})$, time $(\mathrm{Ti})$, season $(\mathrm{Se})$, week $(\mathrm{W})$ and daytime $(\mathrm{Da})$ as categorical variables. Left side correspond to the observed values, right one is equal to the predicted frequencies from the log-linear model 5 .

\begin{tabular}{llrrrrrr}
\hline & & \multicolumn{3}{c}{ Observed values } & \multicolumn{4}{c}{ Fitted values } \\
\hline & & \multicolumn{3}{c}{ type_acc } & \multicolumn{4}{c}{ type_acc } \\
\hline$D, S, T i, S e, D a$ & week & slight_safe & serious & fatal & slight_safe & serious & fatal \\
\hline $\begin{array}{l}\text { Doubs } \\
\text { none }\end{array}$ & weekday & 10 & 27 & 4 & 8,33 & 32,01 & 7,02 \\
7am_10am & week_end & 2 & 8 & 0 & 2,06 & 10,96 & 1,95 \\
spring & & & & & & & \\
day & & & & & & & \\
& & & & & & & \\
\hline$D, S, T i, W, D a$ & season & slight_safe & serious & fatal & slight_safe & serious & fatal \\
\hline $\begin{array}{l}\text { Doubs } \\
\text { none }\end{array}$ & spring & 8 & 36 & 8 & 5,90 & 31,34 & 5,57 \\
11am_3pm & summer & 4 & 44 & 6 & 5,44 & 41,08 & 7,24 \\
week_end & autumn & 5 & 30 & 2 & 3,68 & 30,19 & 6,15 \\
day & winter & 2 & 18 & 4 & 4,52 & 24,26 & 4,87 \\
\hline
\end{tabular}

TAB 6 gives two partial tables of the seven-way contingency table made with type_acc, substance, department, time, season, week et daytime as categorical variables. It is quite difficult, actually, to give the whole seven-way contingency table due to the high number of cells (3840). This table gives frequencies for categories "Doubs", "none", "7am_10am", "spring" and "day" of respectively department, substance, time, season and daytime variables; and "Doubs", "none", "11am_3pm", "week_end" and "day" for respectively department, substance, time, week and daytime variables. The first partial table (top) details according to week and type_acc variables and the second partial table (bottom) details according to season and type_acc variables.

The odds ratios related to the fitted log-linear model from (5) have been calculated 
in the same way as in the previous section and are given in TAB 7. The baseline categories used of type_acc, week and season were respectively "slight_safe", "weekday" and "spring". Here, conditional odds ratios may differ according to which levels of the variables we have conditioned. However there are too many possible combinations of levels with our seven categorical variables used, hence only conditional odds ratios regarding to the fixed levels, as in TAB 7, were calculated. Notice a slight difference between the conditional odds ratios and the marginal ones. However, the interpretations are the same, except for the level "winter" of the variable season for which the odds ratios have a different conclusion depending on whether they are conditional or marginal.

Conditional and marginal odds ratios inform that the risk of an accident being serious or fatal increases if it occurs during the weekend. Indeed, the conditional and marginal odds ratios for an accident to be serious are estimated to be equal to 1,38 on week-ends and respectively 1,12 and 1,34 for an accident being fatal, on week-ends as well. Both kinds of odds ratios tell us that the risk for a crash to be serious or fatal than slightly increases if it occurs in summer or autumn in comparaison with spring. Conditional and marginal odds ratios for an accident to be serious are estimated to be respectively 1,42 and 1,27 if it occurs in summer, 1,54 and 1,37 if it is in autumn. They are estimated to be respectively equal to 1,41 and 1,03 for a fatal crash in summer, 1,77 and 1,23 in autumn. For those occurring during winter with respect to spring, interpretations differ regarding to conditional or marginal odds ratios. Indeed, the risk for an accident to be serious does not seem to depend on the fact that it occurs in winter with respect to spring, the conditional odds ratio being equal to 1,01 . The marginal odds ratio is equal to 0,90 meaning that this risk decreases slightly if it occurs during winter. As for the fatal consequences of an accident occurring in winter, the risk would be slightly increased compared to spring according to the conditional odds ratio equal to 1,14 ; the marginal odds ratio equal to 0,91 informs us that this risk would be slightly reduced.

\subsection{Ordinal regression modelling}

An ordinal regression has been fitted on the response variable type_acc (ordered as slight_safe, serious and then fatal). The analysis was performed with all the explanatory variables except canton (due to too many categories).

Note that the categorical variable type_acc is considered as a response variable. It is relevant because it gives the severity of accidents and the aim of this study lies on understanding how the gendarmerie can avoid serious injuries.

The initial dataset has been split randomly into two sets: a training set (seventy-five percent of the initial one) and a test set (the remaining twenty-five percent). An ordinal regression model has been fitted on the training set, the results are given in TAB 8 . Only five explanatory variables reveal to have an effect on the response variable: time, substance, department, collision and area. This model, with full parameters, gives a misclassification error of $28,84 \%$ on the test set.

Next, a variable selection has been performed by using AIC criterion (with backward selection). The final model is composed by the categorical variables time, substance, department, collision and area. This model gives a misclassification error of $29,00 \%$ on the test set, a score very close to the previous one. The odds ratio of these variables are given in Fig (13). Only odds ratios with confidence intervals not containing the value 1 (represented by the vertical dotted line) are interpreted. 
TAB 7.: Odds ratio estimated from log-linear model 5. The table is divided into two parts, each part is also split up into two parts: conditional odds ratios in top have been calculated in left side controlling the levels of department, substance, time, season and daytime then right side controlling the levels department, substance, time, week and daytime variables, and marginal odds ratios in bottom have been calculated in left side ignoring department, substance, time, season and daytime then in right side ignoring department, substance, time, week and daytime variables. Each odds ratio has been calculated as each level of type_acc, week and season variable and was opposed respectively to "slight_safe", "weekday" and "spring".

\begin{tabular}{|c|c|c|c|c|c|}
\hline \multicolumn{6}{|c|}{ Conditional odds ratios } \\
\hline \multirow{4}{*}{ week_end } & serious & fatal & & serious & fatal \\
\hline & \multirow{3}{*}{1,38} & \multirow{3}{*}{1,12} & summer & 1,42 & 1,41 \\
\hline & & & autumn & 1,54 & 1,77 \\
\hline & & & winter & 1,01 & 1,14 \\
\hline \multicolumn{6}{|c|}{ Marginal odds ratios } \\
\hline \multirow{4}{*}{ week_end } & serious & fatal & & serious & fatal \\
\hline & \multirow{3}{*}{1,38} & \multirow{3}{*}{1,34} & summer & 1,27 & 1,03 \\
\hline & & & autumn & 1,37 & 1,23 \\
\hline & & & winter & 0,90 & 0,91 \\
\hline
\end{tabular}

Regarding the odds ratio, the highest risk for an accident to be serious or fatal is if substances have been consumed by one of the drivers involved. Indeed, the most two important odds ratios are alcohol_drug and drug which are equal to $\exp (1,16)=3,19$ and $\exp (0,85)=2,34$ respectively. The risk for an accident to be serious or fatal increases if the accident happens in non urban areas (odds ratio 2,10) or in the Jura department (odds ratio 1,42 with Doubs as reference). Accident involving uncommon collision also have a slightly increased risk (odds ratio 1,28). On the opposite, two categories have a protective effect and are associated with lower risk of serious or fatal accident. This is the case for Territoire de Belfort department (odds ratio 0,56 with Doubs as reference) and for the occurrence time between $4 \mathrm{pm}$ and $7 \mathrm{pm}$ (odds ratio $0,77)$.

Each department odds ratio has been represented on the map in Fig 14. Remind that the odds of Haute-Saône department were not significant and hence meaningless. The odds ratio of Doubs department is equal to 0 as it was the baseline category for the department variable. As a symbol, Jura and Territoire de Belfort departments have been represented in red and blue respectively due to their odds ratio: the riskiest and the less risky.

\section{Conclusion}

A study of accidents with the purpose of mitigating the crash severity is critical for the well-being of a society and the safety concern posed by road crashes. The aim of this work was to understand factors which are the most influential in road accidents from the French region Franche-Comté. To respond to these issues, three statistical methods were used: Multiple Correspondence Analysis (MCA), log-linear model and ordinal logistic regression. 
TAB 8.: Ordinal regression model results with type_acc as ordered response variable. The item * means that the p-value of the nullity coefficient test is less than 0,05 . The category in parentheses correspond to the baseline category of the above categorical variable.

\begin{tabular}{|c|c|c|c|c|c|c|c|c|c|}
\hline \multirow{2}{*}{ Attribute } & \multicolumn{4}{|c|}{ Ordinal regression results } & \multirow[t]{2}{*}{ Attribute } & \multicolumn{4}{|c|}{ Ordinal regression results } \\
\hline & Coefficients & Estimate & p-value & & & Coefficients & Estimate & $\mathrm{p}$-value & \\
\hline substance & alcohol_drug & 1,16 & $5,19 \mathrm{e}-8$ & $*$ & department & Haute_Saone & $-0,01$ & 0,89 & \\
\hline (none) & drug & 0,85 & $4,32 \mathrm{e}-5$ & $*$ & (Doubs) & Jura & 0,35 & $5,87 \mathrm{e}-5$ & * \\
\hline & alcohol & 0,46 & $1,14 \mathrm{e}-5$ & $*$ & & Terr_Belfort & $-0,58$ & $2,67 \mathrm{e}-4$ & * \\
\hline season & spring & 0,01 & 0,30 & & obstacle & vehicle & $-0,02$ & 0,85 & \\
\hline (winter) & summer & 0,11 & 0,27 & & (none) & pedestrian & 0,23 & 0,15 & \\
\hline & autumn & $-0,05$ & 0,67 & & & other_kind & 0,23 & 0,30 & \\
\hline $\begin{array}{l}\text { week } \\
\text { (weekday) }\end{array}$ & week_end & $-0,04$ & 0,53 & & $\begin{array}{l}\text { shape_road } \\
\text { (straight) }\end{array}$ & curve & $-0,01$ & 0,90 & \\
\hline daytime & day & 0,08 & 0,51 & & collision & usual & $-0,02$ & 0,91 & \\
\hline (night) & & & & & (none) & other_kind & 0,25 & 0,04 & $*$ \\
\hline time & 11am_3pm & $-0,08$ & 0,50 & & type_road & communal & 0,43 & 0,14 & \\
\hline (7am_10am) & $4 \mathrm{pm}_{-} 7 \mathrm{pm}$ & $-0,26$ & 0,02 & $*$ & (highway) & departmental & 0,39 & 0,15 & \\
\hline & $8 \mathrm{pm} \_11 \mathrm{pm}$ & $-0,10$ & 0,52 & & & national & 0,47 & 0,10 & \\
\hline & midnight_6am & 0,18 & 0,27 & & & other_kind & 0,34 & 0,34 & \\
\hline $\begin{array}{l}\text { weather } \\
\text { (normal) }\end{array}$ & other_kind & 0,00 & 0,97 & & $\begin{array}{l}\text { intersection } \\
\text { (out_of_intersection) }\end{array}$ & intersection & $-0,12$ & 0,31 & \\
\hline $\begin{array}{l}\text { area } \\
\text { (urban) }\end{array}$ & unurban & 0,74 & $3,97 \mathrm{e}-16$ & $*$ & & & & & \\
\hline
\end{tabular}

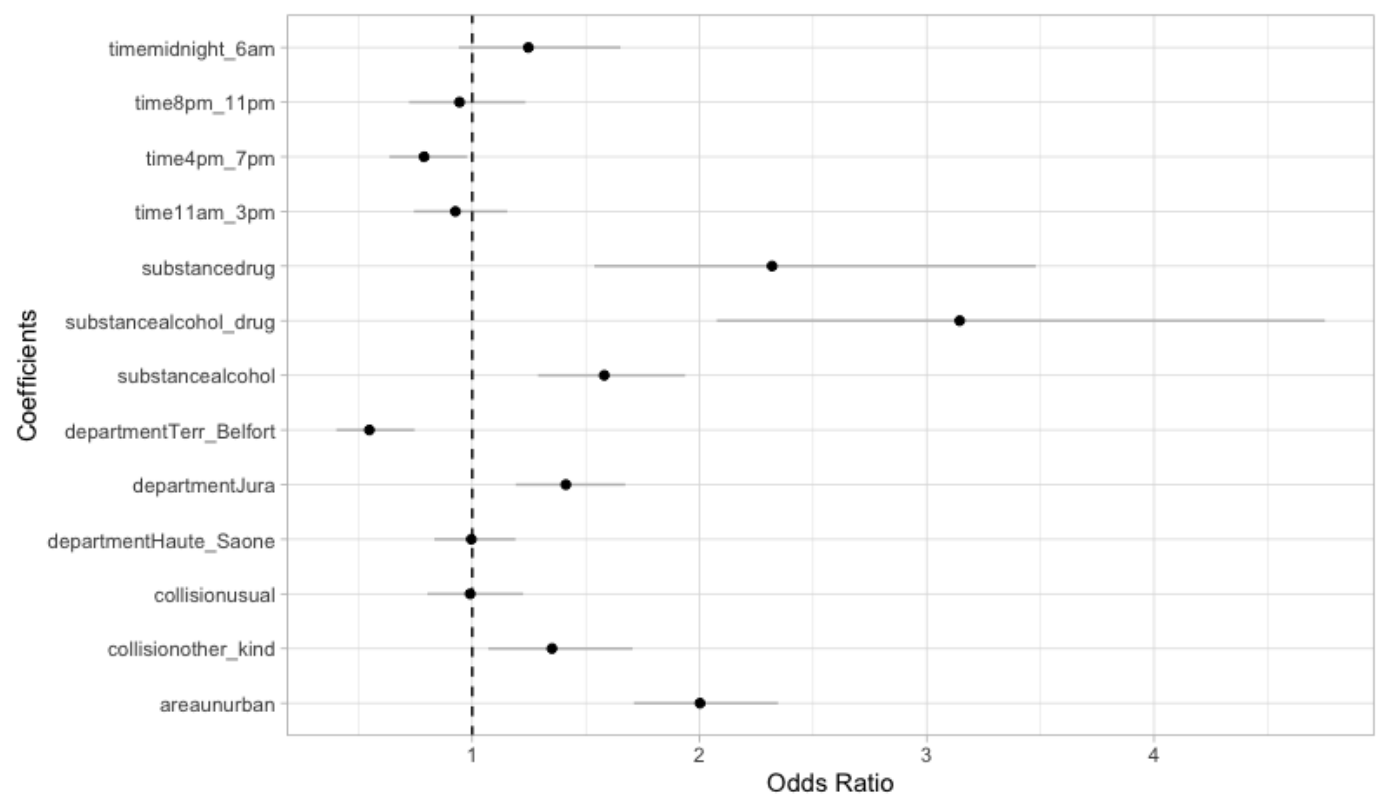

FIG 13.: Odds ratios obtained by ordinal regression model. Grey lines represent the confidence intervals and black points the values of the odds ratios.

MCA, the only unsupervised or descriptive statistical method used in this study, allowed to assess relationships between the categorical variables and examine the associations between the different categories. Geometric representations of data in smaller dimension spaces were produced and proximities between several road crash related 


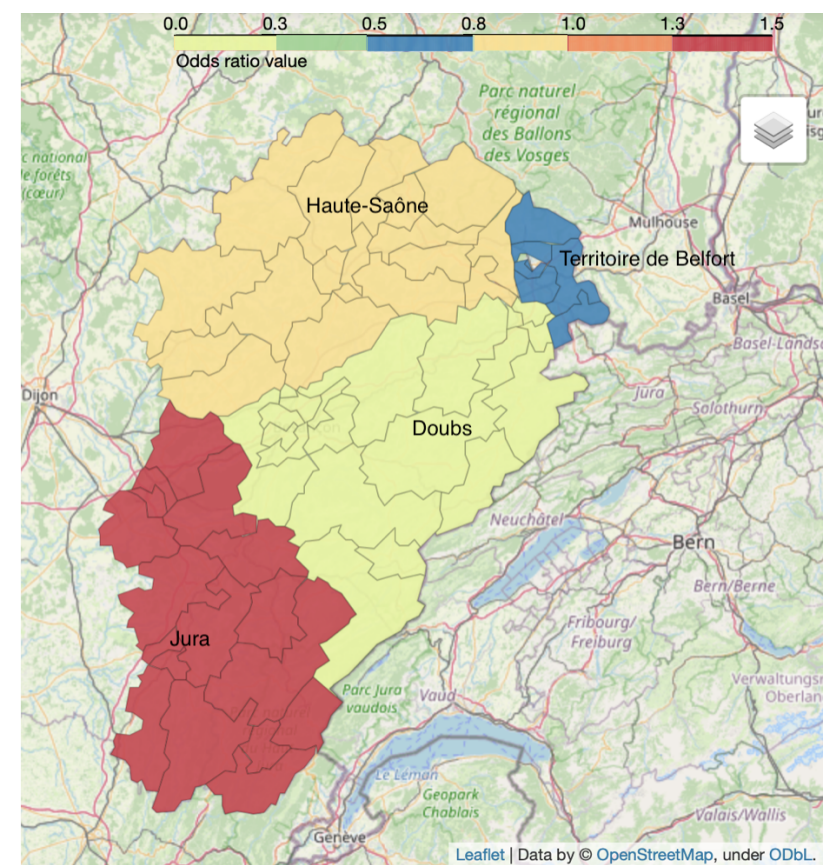

Fig 14.: Franche-Comté map with department odds ratio. Each color corresponds to a department with its odds ratio value considering the Doubs department as the reference and each small division corresponds to a canton.

categories have been observed. This analysis allowed to establish a global vision of the data and to draw up temporal and spatial profiles of accidents occurring in the FrancheComté region. Regarding the MCA temporal analysis, several associations have been highlighted. We remarked that accidents occurring during the week are different from those occurring during the weekend. Indeed, weekend accidents are more likely to happen during the night, be fatal and associated to alcohol/drug consumption. There was also a contrast between summer and winter accidents. The MCA spatial analysis revealed that several cantons of the Franche-Comté region are strongly related to alcohol/drug consumption (Bethoncourt, Saint-Lupicin or Dole) or to fatal accidents (Besançon, Baume-les-Dames, Authume, Saint-Laurent-en-Grandvaux, Saint-Lupicin and Dole).Bethoncourt, Besançon and Baume-les-Dames are situated in the Doubs department and others in the Jura department. Finally, the global MCA temporal and spatial analysis allows to analyse the departments in association to alcohol/drug consumptions and crash severity in temporal ways. Many contrasts have been highlighted and groups of close categories have been analysed. It seems that Jura department is the department where most of accidents are serious or fatal, instead of Territoire de Belfort department which is associated to minor consequences.

The log-linear model was used next in order to evaluate dependencies between the road crash gravity, the alcohol/drug consumption and Franche-Comté departments. This tool models the multidimensional contingency table formed by these three categorical variables and describes associations and interactions among them. It allowed establishing patterns. The selected model concludes on no interaction between the categorical variables type_acc, substance and department. It corresponds to the model of homogeneous association, which means that each pair of variable were conditionally dependent. Odds ratios estimated from this model allowed to quantify the risks about alcohol/drug consumption and the department where the accident happened. 
We remarked that the highest risks for a serious or fatal accident to happen are if drug, alcohol or both are consumed. Conversely, the lowest risk for an accident to be serious or fatal is if it happens in the Territoire de Belfort department. The Jura department was, instead of the previous one, a location which increases this risk. Then, a second log-linear model was fitted, a non-hierarchical one. This model used results from the global MCA. The fairly similar groups of categories were translated into variable interactions which thus induced the fitted log-linear model. This model revealed many associations between variables. Odds ratios estimated from this model allowed to quantify the risk of an accident occurring during the week or the week-end, or occurring in spring, summer, autumn or winter. According to the conditional odds ratios calculated with respect to spring level, the highest risk for an accident to be serious or fatal is if it occurs in autumn.

The ordinal regression allowed the study to assess each effect of road crash related factors on the road crash gravity. Eight circumstances revealed to be influential on the accident gravity: the consumption of alcohol, drug or both; the period of the day between $4 \mathrm{pm}$ and $7 \mathrm{pm}$; the roads situated outside urban areas; the roads situated in Jura or Territoire de Belfort departments; collisions qualified as "other_kind" (not usual as frontal or rear-end for example). Odds ratios estimated from this model allowed to quantify the risks due to each of these circumstances. Similarly to the loglinear analysis, the risk of an accident being fatal is highest if alcohol and drugs are consumed and lowest if the accident happens in the Territoire de Belfort department.

The results obtained with these three methods allow us to conclude that the most important factor to take into account for road crashes in Franche-Comte is the alcohol/drug consumption. As expected, this factor strongly influences the nature of accidents. Hence, based on results obtained with this statistical study, more efforts should be gathered by the National Gendarmerie of Besançon to prevent the alcohol/drug consumption especially in the cantons which were associated to this factor. For example, more alcohol/drug tests and driver awareness measures can be performed.

In order to be more precise for the spatial analysis, the future study would be focused on how GPS coordinate can be used to prevent accidents.

\section{References}

Abdel-Aty, M. A. and Abdelwahab, H. T. (2000). Exploring the relationship between alcohol and the driver characteristics in motor vehicle accidents. Accident Analysis and Prevention, pages 473-482.

Abdel-Aty, M. A., Chen, C., and Schott, J. R. (1998). An assessment of the effect of driver age on traffic accident involvement using log-linear models. Accident Analysis and Prevention, 30(6):851-861.

Agresti, A. (1990). Categorical Data Analysis. Wiley.

Agresti, A. (2013). Categorical Data Analysis. Wiley-Blackwell, 3nd edition.

Andersen, E. (1980). Discrete Statistical Models with Social Science Applications. North-Holland.

Benzécri, J.-P. (1982). Histoire et Préhistoire de l'Analyse des Données. Paris: Dunod.

Benzécri, J.-P. e. a. (1973). L'Analyse des Données: L'Analyse des Correspondences. Paris: Dunod.

Bièvre, D. (2017). Acteurs de contingence et insécurité routière: les limites de la statistique descriptive. Technical report.

Christensen, R. H. B. (2019). ordinal: Regression models for ordinal data. R package 
version 2019.12-10. https://CRAN.R-project.org/package=ordinal.

Das, S., Avelar, R., Dixon, K., and Sun, X. (2018). Investigation on the wrong way driving crash patterns using multiple correspondence analysis. Accident Analysis and Prevention, 11:43-55.

Das, S. and Sun, X. (2015). Factor association with multiple correspondence analysis in vehicle-pedestrian crashes. Trasportation Research Record: Journal of the Transportation Research Board, 2519:95-103.

Das, S. and Sun, X. (2016). Association knowledge for fatal run-off-road crashes by multiple correspondence analysis. IATSS Research, 39:146-155.

Escofier, B. and Pagès, J. (2008). Analyses factorielles simples et multiples. Dunod, 4ème édition edition.

Fort, E., Gadegbeku, B., Gat, E., Pelissier, C., Hours, M., and Charbotel, B. (2019). Working conditions and risk exposure of employees whose occupations require driving on public roads - factorial analysis and classification. Accident Analysis and Prevention, 131:254-267.

Goodman, L. A. (1986). Some useful extensions of the usual correspondence analysis approach and the usual log-linear models approach in the analysis of contingency tables. International Statistical Review, 54:243-309.

Greenacre, M. (1989). The carroll-green-schaffer scaling in correspondence analysis: a theoretical and empirical appraisal. Journal of Marketing Research, 26:358-365.

Greenacre, M. (2006). From simple to multiple correspondence analysis. In Greenacre, M. and Blasius, J., editors, Multiple Correspondence Analysis and Related Methods. Chapman \& Hall/CRC.

Greenacre, M. and Blasius, J. (2006). Multiple Correspondence Analysis and Related Methods. Chapman \& Hall/CRC.

Hothorn, T. and Everitt, B. S. (2014). A Handbook of Statistical Analyses Using R. CRC Press, third edition edition.

Husson, F., Lê, S., and Pagès, J. (2016). Analyse des données avec R. Presses Universitaires de Rennes, 2ème édition edition.

Kassambara, A. and Mundt, F. (2019). factoextra: Extract and Visualize the Results of Multivariate Data Analyses. R package version 1.0.6.

Lê, S., Josse, J., and Husson, F. (2008). FactoMineR: A package for multivariate analysis. Journal of Statistical Software, 25(1):1-18.

Le Roux, B. and Rouanet, H. (2004). Geometric Data Analysis: From Correspondence Analysis to Structured Data. Dordrecth: Kluwer.

McCullagh, P. and Nelder, J. (1989). Generalized Linear Models. Chapman \& Hall/CRC, second edition edition.

Mekonnen, B. (2018). Risk factors of road traffic accidents and its severity in north shewa zone, amhara region, ethiopia. American Journal of Theoretical and Applied Statistics, 7(4):163-166.

ONISR (2019). La sécurité routière en France - Bilan de l'accidentalité de l'année 2018. ONISR, Paris.

Papagiotakos, D. B. and Pitsavos, C. (2004). Interpretation of epidemiological data using multiple correspondence analysis and log-linear models. Journal of Data Science, 2:75-86.

Rezapour, M. and Ksaibati, K. (2018). Application of multinomial and ordinal logistic regression to model injury severity of truck crashes, using violation and crash data. Springer.

Van der Heijden, P., De Falguerolles, A., and De Leeuw, J. (1989). A combined approach to contingency table analysis using correspondence analysis and log-linear 
analysis. Applied Statistics, 38:249-292.

Venables, W. N. and Ripley, B. D. (2002). Modern Applied Statistics with S. Springer, New York, fourth edition. ISBN 0-387-95457-0.

Wickham, H. (2016). ggplot2: Elegant Graphics for Data Analysis. Springer-Verlag New York.

Yannis, G., Golias, J., and Papadimitriou, E. (2005). Driver age and vehicle engine size effects on fault and severity in young motorcyclists accidents. Accident Analysis and Prevention, pages 327-333. 